\title{
LACUNAS IN THE SUPPORT OF THE WEYL CALCULUS FOR TWO HERMITIAN MATRICES
}

\author{
BRIAN JEFFERIES and BERND STRAUB
}

(Received 1 October 2001; revised 17 June 2002)

Communicated by A. Pryde

\begin{abstract}
The connection between Clifford analysis and the Weyl functional calculus for a $d$-tuple of bounded selfadjoint operators is used to prove a geometric condition due to J. Bazer and D. H. Y. Yen for a point to be in the support of the Weyl functional calculus for a pair of hermitian matrices. Examples are exhibited in which the support has gaps.
\end{abstract}

2000 Mathematics subject classification: primary 47A60, 46H30; secondary 47A25, 30G35.

Keywords and phrases: lacunas, Weyl functional calculus, Clifford algebra, monogenic function, symmetric hyperbolic system.

\section{Introduction}

For a $d$-tuple $\mathbf{A}=\left(A_{1}, \ldots, A_{d}\right)$ of noncommuting bounded selfadjoint operators acting on a Hilbert space $H$, there is no direct analogue of the spectral theorem for a single selfadjoint operator $T$, by which a function $f(T)$ of $T$ can be expressed in terms of an integral $f(T)=\int_{\sigma(T)} f(\lambda) d E(\lambda)$ with respect to a spectral measure $E$.

The Weyl functional calculus $\mathscr{W}_{\mathbf{A}}: f \mapsto f_{\mathrm{W}}(\mathbf{A})$ for $\mathbf{A}$ is a means of constructing functions $f_{\mathrm{W}}(\mathbf{A})$ of the system $\mathbf{A}$ of operators, for suitable smooth functions $f$ defined on $\mathbb{R}^{d}$. It was proposed by $\mathrm{H}$. Weyl for the pair $(P, Q)$ of unbounded selfadjoint operators, where $P$ is the momentum operator and $Q$ is the position operator in quantum mechanics. In the noncommuting case, the operator $\mathscr{W}_{\mathbf{A}}(f)$ is not necessarily expressible as an integral with respect to an operator-valued measure, but $\mathscr{W}_{\mathbf{A}}$ is an

This work was supported by an ARC Large Grants at UNSW. We thank S. Okada for thoroughly reading the manuscript and making many helpful criticisms and W. Ricker for useful discussions.

(C) 2003 Australian Mathematical Society 1446-7887/03 \$A2.00+0.00 
operator-valued distribution. If $A$ consists of bounded operators, then $\mathscr{W}_{\mathrm{A}}$ necessarily has compact support.

A feature of Weyl's functional calculus is that $\mathscr{W}_{\mathbf{A}}$ maps a polynomial $p$ in $d$ variables to an operator $p_{\mathrm{W}}(\mathbf{A})$ in which symmetrised products in the elements of $\mathbf{A}$ replace the associated monomial components of $p$.

A similar phenomenon emerges in Clifford analysis. A monogenic function $f$ defined on $\mathbb{R}^{d+1}$, and with values in a finite dimensional Clifford algebra, is a function satisfying a higher dimensional analogue of the Cauchy-Riemann equations. Every complex valued analytic function in $d$ real variables has a unique monogenic extension to an open subset of $\mathbb{R}^{d+1}$. The monogenic extension of polynomials on $\mathbb{R}^{d}$ are the corresponding polynomials in the $d$-tuple $\left(\mathbf{z}_{1}, \ldots, \mathbf{z}_{d}\right)$ of monogenic extensions of coordinate functions, but with products suitably symmetrised.

The purpose of the present work is to exploit the connection between the Weyl and Clifford calculi, previously examined in $[17,18]$, to obtain a geometric expression for the support of the Weyl functional calculus for two hermitian matrices. Expressed otherwise, we describe geometrically the 'joint spectrum' $\gamma(\mathbf{A})$ of two noncommuting hermitian matrices $\mathbf{A}=\left(A_{1}, A_{2}\right)$. In the case that $A_{1}, A_{2}$ do commute, the support $\gamma(\mathbf{A})$ of the Weyl functional calculus $\mathscr{W}_{\mathbf{A}}$ for the pair $\mathbf{A}$ is actually the support of the joint spectral measure for $\mathbf{A}$-the finite set of joint eigenvalues of $A_{1}$ and $A_{2}$. Our technique uses a generalisation of the Cauchy-Stieltjes transform of a measure on $\mathbb{R}$ from the complex plane to higher dimensions. The analogy is as follows.

Let $\mu$ be a finite Borel measure on the line. The Cauchy-Stieltjes transform $\tilde{\mu}$ is defined for all $z \in \mathbb{C} \backslash \operatorname{supp} \mu$ by

$$
\tilde{\mu}(z)=\frac{1}{2 \pi i} \int_{\mathbb{R}} \frac{d \mu(x)}{z-x} .
$$

The measure $\mu$ can be recovered from its Cauchy-Stieltjes transform $\tilde{\mu}$ by the formula

$$
\int_{\mathbb{R}} \phi d \mu=\lim _{\varepsilon \rightarrow 0^{+}} \int_{\mathbb{R}}[\tilde{\mu}(x-i \varepsilon)-\tilde{\mu}(x+i \varepsilon)] \phi(x) d x,
$$

valid for all smooth functions $\phi$ with compact support.

A similar argument applies to a spectral measure. Suppose that $A$ is a selfadjoint operator acting in a Hilbert space with a selfadjoint spectral measure $P$ supported on the spectrum $\sigma(A)$ of $A$, that is, $A=\int_{\sigma(A)} \lambda d P(\lambda)$. Then for every $z$ belonging to the resolvent set $\rho(A)=\mathbb{C} \backslash \sigma(A)$, the functional calculus for selfadjoint operators gives

$$
\tilde{P}(z)=\frac{1}{2 \pi i} \int_{\mathbb{R}} \frac{d P(x)}{z-x}=\frac{1}{2 \pi i}(z I-A)^{-1}
$$

in terms of the resolvent $(z I-A)^{-1}$ of $A$. Moreover,

$$
\int_{\mathbb{R}} \phi d P=\frac{1}{2 \pi i} \lim _{\varepsilon \rightarrow 0^{+}} \int_{\mathbb{R}}\left[((x-i \varepsilon) I-A)^{-1}-((x+i \varepsilon) I-A)^{-1}\right] \phi(x) d x
$$


for all smooth functions $\phi$ with compact support (see [11, (iv) page 2168]). The support $\sigma(A)$ of the spectral measure $P$ is characterised as the complement of the set of points $x \in \mathbb{R}$ contained in an open set $U$ in $\mathbb{C}$ in which the resolvent $z \mapsto(z I-A)^{-1}$, $z \in U \backslash \mathbb{R}$, is the restriction to $U \backslash \mathbb{R}$ of a continuous function defined in $U$. Formulas like (1) are basic to the spectral theory of differential operators and to the construction of their spectral measures, for example, by the Weyl-Titchmarsh-Kodaira formula for Sturm-Liouville differential operators.

In the case that $\mathbf{A}=\left(A_{1}, \ldots, A_{d}\right)$ is a $d$-tuple of bounded selfadjoint operators acting on a Hilbert space $H$ and $\mathscr{W}_{\mathbf{A}}$ is the Weyl functional calculus associated with A, the equation

$$
\mathscr{W}_{\mathbf{A}}(\phi)=\lim _{\varepsilon \rightarrow 0^{+}} \int_{\mathbb{R}^{d}}\left[G_{x+\varepsilon e_{0}}(\mathbf{A})-G_{x-\varepsilon e_{0}}(\mathbf{A})\right] \phi(x) d x, \quad \phi \in C_{c}^{\infty}\left(\mathbb{R}^{d}\right),
$$

was established in [17, Theorem 6.2]. Here $G_{\omega}(\mathbf{A}), \omega \in \mathbb{R}^{d+1} \backslash\left(\{0\} \times \operatorname{supp}\left(\mathscr{W}_{\mathbf{A}}\right)\right)$, is a higher dimensional analogue of the resolvent family of a single operator. The Cauchy kernel $G_{(\cdot)}(\mathrm{A})$ takes values in a Clifford module over the space of bounded linear operators acting on $H$. It can be viewed as a higher dimensional Cauchy-Stieltjes transform of the distribution $\mathscr{W}_{\mathbf{A}}$ supported in $\mathbb{R}^{d}$. The analogue

$$
f_{\mathrm{W}}(\mathbf{A})=\int_{\partial \Omega} G_{\omega}(\mathbf{A}) n(\omega) f(\omega) d \mu(\omega)
$$

of the Riesz-Dunford formula is also valid for functions $f$ left monogenic in a neighbourhood in $\mathbb{R}^{d+1}$ of the support of $\mathscr{W}_{\mathbf{A}}[17$, Corollary 5.5].

Then $\operatorname{supp}\left(\mathscr{W}_{\mathbf{A}}\right)$ is characterised as the complement of the set on which the function $\omega \mapsto G_{\omega}(\mathbf{A})$ is continuous, that is, the 'spectrum' $\operatorname{supp}\left(\mathscr{W}_{\mathbf{A}}\right)$ of the functional calculus $\mathscr{W}_{\mathrm{A}}$ is precisely the set of singularities of the Cauchy kernel $G_{(\cdot)}(\mathrm{A})$, just as the spectrum $\sigma(T)$ of a single operator $T$ is the set of singularities of the resolvent $\lambda \mapsto(\lambda I-T)^{-1}$. Although the Fourier transform $\widehat{\mathscr{W}}_{\mathrm{A}}$ of the operator-valued distribution $\mathscr{W}_{\mathrm{A}}$ is known explicitly, it does not obviously provide detailed information about the local behaviour of $\mathscr{W}_{\mathbf{A}}$. For example, in the case of a pair $\mathbf{A}=\left(A_{1}, A_{2}\right)$ of bounded selfadjoint operators, an application of the Paley-Wiener theorem shows that the convex hull of $\operatorname{supp}\left(\mathscr{W}_{\mathbf{A}}\right)$ coincides with the closure of the numerical range of the bounded linear operator $A_{1}+i A_{2}$ [2, Theorem 5.2]. It is difficult to obtain further information from bounds involving the Fourier transform $\widehat{\mathscr{W}}_{\mathbf{A}}$ of $\mathscr{W}_{\mathbf{A}}$.

A simple geometric condition for points to belong to $\operatorname{supp}\left(\mathscr{W}_{\mathbf{A}}\right)$ in the case that $\mathbf{A}=\left(A_{1}, A_{2}\right)$ is a pair of hermitian matrices is found in Theorem 6.4 from the plane wave decomposition for $G_{\omega}(\mathbf{A})$ [18, Lemma 2.5], as this is adapted to a more detailed examination of the behaviour of $\mathscr{W}_{\mathbf{A}}$ around its support.

The argument we use demonstrates why Clifford analysis or, more specifically, quaternionic analysis is more suited to the problem at hand than the theory of functions 
of several complex variables. The distribution $\mathscr{W}_{\mathbf{A}}$ is supported in $\mathbb{R}^{2}$ and can $b$ represented as the boundary values of a function, taking its values in a Clifford moduls over the space of matrices, and monogenic in $\mathbb{R}^{3}$ off the plane $\mathbb{R}^{2} \equiv\{0\} \times \mathbb{R}^{2} \subset \mathbb{R}^{3}$ However, if we represent the distribution $\mathscr{W}_{\mathbf{A}}$ as the boundary values on $\mathbb{R}^{2}$ of : matrix-valued analytic function $\Phi$ defined in $\mathbb{C}^{2}$, then we find that $\Phi$ has singularitie on certain algebraic curves studied by Kippenhahn [23] in his 1951 investigation intc the numerical range of matrices; this phenomenon complicates the analysis. The emergence of singularities in $\mathbb{C}^{n}$ in the Cauchy transform of smooth scalar-valuec functions in $\mathbb{R}^{n}$ for $n>1$ is noted in [10].

The distribution $(x, t) \mapsto \mathscr{W}_{t \mathrm{~A}}(x)$ is actually the fundamental solution of a linea symmetric hyperbolic system of PDE of a type that arises, for example, in the study of two-dimensional magnetohydrodynamic waves [35]. Part of Theorem 6.4 has beer obtained by Bazer and Yen $[6,7]$ by appealing to a plane wave decomposition for $\mathscr{W}_{t A}$ from the method of Herglotz and Radon [20], although no connection is made in these works with Kippenhahn's characterisation [23] of the numerical range of matrices Our approach also makes explicit the relationship with the 'spectral theory' of a finite collection of noncommuting operators.

A more general study of the support of the fundamental solution of hyperbolic PDE with constant coefficients originated with the penetrating work of Petrovsky [27] anc was advanced by Atiyah, Bott and Gårding [3, 4], see also [34]. The lacunas studiec in the present work and in $[27,6,7]$ are called strong lacunas in the terminology of [3] and [4]. The fundamental solution of a symmetric hyperbolic system $P$ of PDE may have lacunas stronger than those of det $P$ [3, page 188]. As in the works [6,7] we use the additional features of symmetric hyperbolic systems to obtain results more directly than those obtained from the general theory of Atiyah, Bott and Gårding $[3,4]$. In particular, the formulas of Herglotz-Petrovsky-Leray [3, Theorem 7.16] for the fundamental solution of det $P$ are replaced in the present context by a much simples formula of Bazer and Yen for the matrix valued distribution $\mathscr{W}_{\mathrm{IA}}$, see Lemma 9.2.

The method of the present work demonstrates that the monogenic functional calculus for noncommuting systems of operators is a useful tool to analyse the suppor of distributions in $\mathbb{R}^{d}$. It also makes explicit the connection between earlier work of Kippenhahn [23] and Murnaghan [25] on the numerical range $W(A)$ of the matrix $A=A_{1}+i A_{2}$ and the support of the Weyl functional calculus $\mathscr{W}_{\mathbf{A}}$ for a pair $\mathbf{A}=\left(A_{1}, A_{2}\right)$ of hermitian matrices. In particular, lacunas or gaps in the support of $\mathscr{W}_{\mathrm{A}}$-the difference between the convex set $W(A)$ and $\operatorname{supp}\left(\mathscr{W}_{\mathrm{A}}\right)$-are already explicit in the numerical range of certain $(3 \times 3)$ matrices $A$ exhibited in [23], see Figures 1-6.

An outline of Clifford analysis is given in Sections 2-3. The higher-dimensional analogue of the Riesz-Dunford functional calculus is outlined in Section 4. An elementary proof of the plane wave decomposition for the Cauchy kernel in $\mathbb{R}^{3}$ suitable for the present purpose is given in Section 5. 
The geometric condition characterising the monogenic spectrum $\gamma(\mathbf{A})$ and the support of the Weyl functional calculus is introduced in Section 6. The remainder of the work is devoted to a proof of Theorem 6.4 using spectral theory arguments for systems of operators.

Our arguments from spectral theory have mainly a functional analytic flavour, but certain geometric ideas, already evident in the pioneering work of Petrovsky [27], are needed to implement the analysis. We include a brief discussion of algebraic curves in Section 7 in an attempt to alleviate the reader's burden with possibly unfamiliar ideas already introduced. Some concrete examples of Kippenhahn's plane algebraic curves for $(3 \times 3)$ matrices are exhibited in Section 8. The proof of Theorem 6.4 is completed in Section 9.

Finally, in Section 10, we make some suggestions about what extensions to the arguments advanced may be valid for finite systems $\mathbf{A}=\left(A_{1}, \ldots, A_{d}\right)$ of matrices, or bounded linear operators on a Banach space for which the spectrum $\sigma\left(\sum_{j=1}^{d} A_{j} \xi_{j}\right)$ of the operator $\sum_{j=1}^{d} A_{j} \xi_{j}$ is a subset of the real numbers for every $\xi \in \mathbb{R}^{d}$.

\section{Clifford algebras}

Let $\mathbb{F}$ be either the field $\mathbb{R}$ of real numbers or the field $\mathbb{C}$ of complex numbers. The Clifford algebra $\mathbb{F}_{(d)}$ over $\mathbb{F}$ is a $2^{d}$-dimensional algebra with unit generated by the standard basis vectors $e_{0}, e_{1}, \ldots, e_{d}$ of the vector space $\mathbb{F}^{d+1}$. Multiplication in $\mathbb{F}_{(d)}$ satisfies $e_{j} e_{k}+e_{k} e_{j}=-2 \delta_{j k}$ for $1 \leq j, k \leq d$, with $\delta_{j k}$ denoting the Kronecker delta. The vector $e_{0}$ serves as the identity element.

A basis of $\mathbb{F}_{(d)}$ is given by vectors $e_{S}$, indexed by all subsets $S$ of the finite set $\{1, \ldots, d\}$. For each such subset $S$, the element $e_{S}$ is the ordered product of the vectors $e_{j}$ with $j \in S$, with the understanding that $e_{\emptyset}=e_{0}$.

The Clifford algebras $\mathbb{R}_{(0)}, \mathbb{R}_{(1)}$ and $\mathbb{R}_{(2)}$ are the real, complex numbers and the quaternions, respectively.

The conjugate $\overline{e_{S}}$ of a basis element $e_{S}$ is defined so that $e_{S} \overline{e_{S}}=\overline{e_{S}} e_{S}=1$. Denote the complex conjugate of a number $c \in \mathbb{F}$ by $\bar{c}$. Then the operation of conjugation $u \mapsto \bar{u}$ defined by $\bar{u}=\sum_{S} \overline{u_{S}} \overline{e_{S}}$ for every $u=\sum_{S} u_{S} e_{S}, u_{S} \in \mathbb{F}$ is an involution of the Clifford algebra $\mathbb{F}_{(d)}$. Then $\bar{u} \bar{v}=\overline{v u}$ for all elements $u$ and $v$ of $\mathbb{F}_{(d)}$. An inner product is defined on $\mathbb{F}_{(d)}$ by the formula $(u, v)=[u \bar{v}]_{0}=\sum_{S} u_{S} \overline{v_{S}}$ for every $u=\sum_{S} u_{S} e_{S}$ and $v=\sum_{S} v_{S} e_{S}$ belonging to $\mathbb{F}_{(d)}$. The corresponding norm is written as $\mid \cdot 1$.

The Clifford algebra $\mathbb{F}_{(d)}$ has the appealing property that any nonzero vector $x \in$ $\mathbb{R}^{d+1}$ has an inverse $x^{-1}$ in the algebra given by $x^{-1}=\bar{x} /|x|^{2}$.

The algebraic tensor product $X_{(d)}=X \otimes \mathbb{F}_{(d)}$ of a vector space $X$ over $\mathbb{F}$ with $\mathbb{F}_{(d)}$ is a two-sided module. Elements of $X_{(d)}$ may be viewed as finite sums $u=\sum_{S} x_{S} e_{S}$ 
of tensor products of elements $x_{S}$ of $X$ with basis vectors $e_{S}$ of $\mathbb{F}_{(d)}$. Multiplication in $X_{(d)}$ by elements $\lambda$ of the Clifford algebra $\mathbb{F}_{(d)}$ is defined by $u \lambda=\sum_{s} x_{S}\left(e_{S} \lambda\right)$ and $\lambda u=\sum_{s} x_{S}\left(\lambda e_{S}\right)$. If $X$ is a normed space, then the norm on $X_{(d)}$ is taken to be $\|u\|=\left(\sum_{S}\left\|x_{S}\right\|_{X}^{2}\right)^{1 / 2}$.

Let $X$ be a Banach space and $\mathscr{L}(X)$ the space of continuous linear operators acting on $X$. The space $\mathscr{L}(X)_{(d)}$ and the space $\mathscr{L}_{(d)}\left(X_{(d)}\right)$ of all right module homomorphisms of $X_{(d)}$ are identified by defining the operation of $T=\sum_{S} T_{S} e_{S}$ on $u=\sum_{S} u_{S} e_{S}$ as $T(u)=\sum_{S, S^{\prime}} T_{S}\left(u_{S^{\prime}}\right) e_{S} e_{S^{\prime}}$. The norm of $T$ is given by $\|T\|=\left(\sum_{S}\left\|T_{S}\right\|_{\mathscr{L}(X)}^{2}\right)^{1 / 2}$. The space $\mathscr{L}(X)$ is identified with the subspace of $\mathscr{L}(X)_{(d)}$ consisting of all elements $T e_{0}, T \in \mathscr{L}(X)$. An element $T$ of $\mathscr{L}(X)_{(d)}$ has an inverse $S$ if $S T=T S=1 e_{0}$. If such an inverse exists, it is written as $T^{-1}$.

\section{Clifford analysis}

What is usually called Clifford analysis is the study of functions of finitely many real variables, which take values in a Clifford algebra, and which satisfy higher dimensional analogues of the Cauchy-Riemann equations.

A function $f: U \rightarrow \mathbb{F}_{(d)}$ defined in an open subset $U$ of $\mathbb{R}^{d+1}$ has a unique representation $f=\sum_{S} f_{S} e_{S}$ in terms of $\mathbb{F}$-valued functions $f_{S}, S \subseteq\{1, \ldots, d\}$ in the sense that $f(x)=\sum_{S} f_{S}(x) e_{S}$ for all $x \in U$. Then $f$ is continuous, differentiable and so on, in the normed space $\mathbb{F}_{(d)}$, if and only if for all finite subsets $S$ of $\{1, \ldots, d\}$, its scalar component functions $f_{S}$ have the corresponding property. Let $\partial_{j}$ be the operator of differentiation of a scalar function in the $j$ th coordinate in $\mathbb{R}^{d+1}$-the coordinates of $x \in \mathbb{R}^{d+1}$ are written as $x=\left(x_{0}, x_{1}, \ldots, x_{d}\right)$. For a continuously differentiable function $f: U \rightarrow \mathbb{F}_{(d)}$ defined in an open subset $U$ of $\mathbb{R}^{d+1}$ with $f=\sum_{s} f_{s} e_{s}$, the function $D f$ is defined by setting $D f=\sum_{s} \sum_{j=0}^{d}\left(\partial_{j} f_{S}\right) e_{j} e_{S}$ and $f D$ is specified by $f D=\sum_{s} \sum_{j=0}^{d}\left(\partial_{j} f_{s}\right) e_{s} e_{j}$.

Now suppose that $f$ is an $\mathbb{F}_{(d)}$-valued, continuously differentiable function defined in an open subset $U$ of $\mathbb{R}^{d+1}$. Then $f$ is said to be left monogenic in $U$ if $D f(x)=0$ for all $x \in U$ and right monogenic in $U$ if $f D(x)=0$ for all $x \in U$. The definition extends to functions $f$ with values in a Clifford module over $\mathbb{F}_{(d)}$.

For each $y \in \mathbb{R}^{d+1}$, the function $G_{y}: \mathbb{R}^{d+1} \backslash\{y\} \rightarrow \mathbb{F}_{(d)}$ defined by

$$
x \mapsto G_{y}(x)=\frac{1}{\sigma_{d}} \frac{\overline{y-x}}{|y-x|^{d+1}},
$$

for all $x \neq y$ is both left and right monogenic. Here the volume of the unit $d$-sphere in $\mathbb{R}^{d+1}$ has been denoted by $\sigma_{d}$ and $\mathbb{R}^{d+1}$ is identified with a subspace of $\mathbb{F}_{(d)}$. In [9], the notation $E(y-x)=G_{y}(x)$ for $y \neq x$ is used. In the present work, we replace $x$ by a $d$-tuple $\mathbf{A}$ of matrices or operators, so the given notation is more convenient. 
The function (2) plays a special role in Clifford analysis. Suppose that $\Omega \subset \mathbb{R}^{d+1}$ is a bounded open set with smooth boundary $\partial \Omega$ and exterior unit normal $n(\omega)$ defined for all $\omega \in \partial \Omega$. For any left monogenic function $f$ defined in a neighbourhood $U$ of $\bar{\Omega}$, the Cauchy integral formula

$$
\int_{\partial \Omega} G_{\omega}(x) n(\omega) f(\omega) d \mu(\omega)= \begin{cases}f(x), & \text { if } \quad x \in \Omega ; \\ 0, & \text { if } \quad x \in U \backslash \bar{\Omega}\end{cases}
$$

is valid. Here $\mu$ is the surface measure of $\partial \Omega$. The result is proved in [9, Corollary 9.6]. If $g$ is right monogenic in $U$ then $\int_{\partial \Omega} g(\omega) n(\omega) f(\omega) d \mu(\omega)=0$ [9, Corollary 9.3].

\section{The monogenic calculus}

Suppose that $\mathbf{A}=\left(A_{1}, \ldots, A_{d}\right)$ is a $d$-tuple of hermitian operators acting on a Hilbert space $H$. For each $\xi \in \mathbb{R}^{d}$, set $\langle\mathbf{A}, \xi\rangle=\sum_{j=1}^{d} A_{j} \xi_{j}$.

The Weyl functional calculus $[2,26,33]$ is a means of forming functions $f_{\mathrm{W}}\left(A_{1}\right.$, $\left.\ldots, A_{d}\right)$ of the $d$-tuple $\mathbf{A}=\left(A_{1}, \ldots, A_{d}\right)$ of operators. There exists a unique operator valued distribution $\mathscr{W}_{\mathbf{A}}: f \mapsto f_{\mathrm{W}}(\mathbf{A}), f \in C^{\infty}\left(\mathbb{R}^{d}\right)$, defined over the test function space $C^{\infty}\left(\mathbb{R}^{d}\right)$ of all infinitely differentiable functions, such that the restriction of $\mathscr{W}_{\mathbf{A}}$ to $\mathscr{S}\left(\mathbb{R}^{d}\right)$ is given by

$$
\mathscr{W}_{\mathbf{A}}(f)=(2 \pi)^{-d} \int_{\mathbb{R}^{d}} e^{i\langle\mathbf{A}, \xi\rangle} \hat{f}(\xi) d \xi
$$

The integral converges as a Bochner integral in $\mathscr{L}(H)$ with the operator norm. The support $\operatorname{supp}\left(\mathscr{W}_{\mathbf{A}}\right)$ of this distribution is contained in the closed ball in $\mathbb{R}^{d}$ centred at zero and with radius $r=\left(\sum_{j=1}^{d}\left\|A_{j}\right\|^{2}\right)^{1 / 2}[33$, Theorem 1].

By virtue of the standard techniques of distribution theory, the distribution $\mathscr{W}_{\mathrm{A}}$ also applies to any smooth function $f: U \rightarrow \mathbb{F}_{(d)}$ defined in an open neighbourhood $U$ of $\operatorname{supp}\left(\mathscr{W}_{\mathbf{A}}\right)$ in $\mathbb{R}^{d+1}$, by applying $\mathscr{W}_{\mathbf{A}}$ to each of the components of $f$ restricted to the open neighbourhood $U \cap \mathbb{R}^{d}$ of $\operatorname{supp}\left(\mathscr{W}_{\mathbf{A}}\right)$ in $\mathbb{R}^{d}$.

Then the $\mathscr{L}(H)_{(d)}$-valued function $\omega \mapsto G_{\omega}(\mathbf{A})$ defined by

$$
G_{\omega}(\mathbf{A})=\mathscr{W}_{\mathbf{A}}\left(G_{\omega}\right), \quad \text { for all } \omega \in \mathbb{R}^{d+1} \backslash\left(\{0\} \times \operatorname{supp}\left(\mathscr{W}_{\mathbf{A}}\right)\right),
$$

is called the Cauchy kernel for the $d$-tuple A. It is the Cauchy-Stieltjes transform of the operator-valued distribution $\mathscr{W}_{\mathbf{A}}$ in the sense of Clifford analysis [9, Definition 27.6].

By an elementary argument [17, Corollary 5.5], the Cauchy integral formula (3) ensures that

$$
\mathscr{W}_{\mathbf{A}}(f)=\int_{\partial \Omega} G_{\omega}(\mathbf{A}) n(\omega) f(\omega) d \mu(\omega)
$$


for any function $f$ left monogenic in a neighbourhood of the closure of the region $\Omega$ in $\mathbb{R}^{d+1}$ containing the support $\operatorname{supp}\left(\mathscr{W}_{\mathbf{A}}\right)$ of $\mathscr{W}_{\mathbf{A}}$ in $\mathbb{R}^{d} \equiv\{0\} \times \mathbb{R}^{d}$.

The monogenic spectrum of $\mathbf{A}$ is the subset $\gamma(\mathbf{A})$ of $\mathbb{R}^{d}$ off which the function

$$
\omega \mapsto G_{\omega}(\mathbf{A}), \quad \omega \in \mathbb{R}^{d+1} \backslash(\{0\} \times \gamma(\mathbf{A}))
$$

is a left and right monogenic function. It is proved in [17] that the set $\gamma(\mathbf{A})$ is precisely the support $\operatorname{supp}\left(\mathscr{W}_{\mathbf{A}}\right)$ of the distribution $\mathscr{W}_{\mathbf{A}}$.

For example, any analytic function $f$ of $d$ real variables defined in an open neighbourhood $U$ of $\gamma(\mathbf{A})$ in $\mathbb{R}^{d}$ is the restriction to $U$ of a left monogenic function $\hat{f}$ defined in an open subset of $\mathbb{R}^{d+1}[9$, Proposition 14.4]. Then formula (6) defines the operator $f(\mathbf{A}):=\tilde{f}(\mathbf{A})$ in such a way that for any polynomial $p$ in $d$ real variables, the operator $p(\mathbf{A})$ is formed from $p$ by replacing terms $x_{j_{1}} \cdots x_{j_{k}}$ by symmetric products in the bounded linear operators $A_{j_{1}}, \ldots, A_{j_{k}}$.

\section{The plane wave decomposition of the Cauchy kernel}

We now restrict ourselves to the case where $d=2$ and $\mathbf{A}=\left(A_{1}, A_{2}\right)$ is a pair of hermitian operators in a finite dimensional Hilbert space.

Throughout the following, $\mathbb{T}$ denotes the unit circle centred at zero in $\mathbb{R}^{2}$ and $\mu$ is the arc length measure of $\mathbb{T}$. The inverse in the integrand of the following formula is understood as an inverse in the Clifford algebra $\mathbb{R}_{(2)}$.

The following elementary calculation is a special case of a general formula [32, page 111], [30, 24] for the plane wave decomposition of the Cauchy kernel in $\mathbb{R}^{d+1}$.

LEMMA 5.1. Let $y \in \mathbb{R}^{2}$ and suppose that $y_{0} \in \mathbb{R}$ is nonzero. Then

$$
\frac{y_{0}-y}{\left(y_{0}^{2}+|y|^{2}\right)^{3 / 2}}= \begin{cases}-\frac{1}{2 \pi} \int_{\pi}\left(\langle y, s\rangle-y_{0} t\right)^{-2} d \mu(t) & y_{0}>0 ; \\ \frac{1}{2 \pi} \int_{\pi}\left(\langle y, t\rangle-y_{0} t\right)^{-2} d \mu(t) & y_{0}<0 .\end{cases}
$$

PROOF. Let us calculate

$$
\int_{\mathbb{\pi}}\left(\langle y, t\rangle-y_{0} t\right)^{-2} d \mu(t)=\int_{\mathbb{J}}\left(\frac{\langle y, t\rangle+y_{0} t}{\langle y, t\rangle^{2}+y_{0}^{2}}\right)^{2} d \mu(t)
$$

for nonzero $y \in \mathbb{R}^{2}$ and $y_{0} \in \mathbb{R}$. Choose coordinates such that $\langle y, t\rangle=|y| \cos \theta$. Then (7) is equal to

$$
\int_{0}^{2 \pi} \frac{|y|^{2} \cos ^{2} \theta-y_{0}^{2}+2 y_{0} y \cos ^{2} \theta}{\left(|y|^{2} \cos ^{2} \theta+y_{0}^{2}\right)^{2}} d \theta .
$$


The term involving $\sin \theta$ has integral zero, so it does not contribute to the integral (8). Because

we have

$$
\begin{aligned}
& \int_{0}^{2 \pi} \frac{\cos ^{2} \theta}{\left(a^{2} \cos ^{2} \theta+b^{2}\right)^{2}} d \theta=\frac{\pi}{|b|\left(a^{2}+b^{2}\right)^{3 / 2}}, \\
& \int_{0}^{2 \pi} \frac{1}{\left(a^{2} \cos ^{2} \theta+b^{2}\right)^{2}} d \theta=\frac{\pi\left(a^{2}+2 b^{2}\right)}{|b|^{3}\left(a^{2}+b^{2}\right)^{3 / 2}}
\end{aligned}
$$

$$
2 y_{0} y \int_{0}^{2 \pi} \frac{\cos ^{2} \theta}{\left(|y|^{2} \cos ^{2} \theta+y_{0}^{2}\right)^{2}} d \theta=\frac{2 \pi y}{\left(|y|^{2}+y_{0}^{2}\right)^{3 / 2}} \operatorname{sgn} y_{0}
$$

and

$$
\int_{0}^{2 \pi} \frac{|y|^{2} \cos ^{2} \theta-y_{0}^{2}}{\left(|y|^{2} \cos ^{2} \theta+y_{0}^{2}\right)^{2}} d \theta=-\frac{2 \pi\left|y_{0}\right|}{\left(|y|^{2}+y_{0}^{2}\right)^{3 / 2}} .
$$

The plane wave representation of the Cauchy kernel $G_{\omega}(\mathrm{A})$ given next was proved in [18] for $d$-tuples of operators. It is critical for the subsequent calculations. The inverse in the integrand is taken in the Clifford module $\mathscr{L}\left(\mathbb{C}^{n}\right)_{(2)}$.

THEOREM 5.2. Let $\mathbf{A}=\left(A_{1}, A_{2}\right)$ be two $(n \times n)$ hermitian matrices. Then for $y \in \mathbb{R}^{2}$ and $y_{0} \neq 0$, the Cauchy kernel $G_{y+y_{0} e_{0}}(\mathbf{A})=\mathscr{W}_{\mathbf{A}}\left(G_{y+y_{0} e_{0}}\right)$ admits the representation

$$
G_{y+y_{0} e_{0}}(\mathbf{A})=-\frac{\operatorname{sgn} y_{0}}{8 \pi^{2}} \int_{\mathbb{T}}\left(\langle y I-\mathbf{A}, t\rangle-y_{0} t I\right)^{-2} d \mu(t) .
$$

PROOF. According to Lemma 5.1, if $y_{0} \neq 0$ and $x \in \mathbb{R}^{3}$ is not equal to $y+y_{0} e_{0}$, then

$$
G_{y+y_{0} e_{0}}(x)=-\frac{\operatorname{sgn} y_{0}}{8 \pi^{2}} \int_{\mathbb{T}}\left(\langle y-x, t\rangle-y_{0} s\right)^{-2} d \mu(t) .
$$

The function $x \mapsto G_{y+y_{0} e_{0}}(x)$ is $C^{\infty}$ for $x \in \mathbb{R}^{3} \backslash\left\{y+y_{0} e_{0}\right\}$, so if $y_{0} \neq 0$, then we have

$$
\begin{aligned}
G_{y+y_{0} e_{0}}(\mathbf{A})=\mathscr{W}_{\mathbf{A}}\left(G_{y+y_{0} e_{0}}\right) & =-\frac{\operatorname{sgn} y_{0}}{8 \pi^{2}} \mathscr{W}_{\mathbf{A}}\left(\int_{\mathbb{T}}\left(\langle y-\cdot, t\rangle-y_{0} t\right)^{-2} d \mu(t)\right) \\
& =-\frac{\operatorname{sgn} y_{0}}{8 \pi^{2}} \int_{\mathbb{T}} \mathscr{W}_{\mathbf{A}}\left(\langle y-\cdot, t\rangle-y_{0} t\right)^{-2} d \mu(t) \\
& =-\frac{\operatorname{sgn} y_{0}}{8 \pi^{2}} \int_{\mathbb{I}}\left(\langle y I-\mathbf{A}, t\rangle-y_{0} t I\right)^{-2} d \mu(t) .
\end{aligned}
$$

The first of the equalities above is the definition (5) of $G_{y+y_{0} e_{0}}(\mathbf{A})$. The second follows from Lemma 5.1. The continuous linear operator $\mathscr{W}_{\mathrm{A}}$ can be passed from 
outside the integral to inside the integral by appealing to a property of Bochner integrals (see [17]).

The last equality can be seen from the equality $\mathscr{W}_{\mathbf{A}}(p(\langle\cdot, \xi\rangle))=p(\langle\mathbf{A}, \xi\rangle)$, valid for any polynomial $p$ on $\mathbb{C}$ and any $\xi \in \mathbb{R}^{2}$. An appeal to Runge's theorem and continuity shows that $p$ can be replaced by any complex function $f$ which is analytic on the compact subset $\left\langle\operatorname{supp}\left(\mathscr{W}_{\mathbf{A}}\right), \xi\right\rangle$ of $\mathbb{R}$. In particular, for each $t \in \mathbb{T}, y_{0} \neq 0$ and $y \in \mathbb{R}^{2}$, the $\mathbb{R}_{(2)}$-valued function $f_{t}: x \mapsto\left(\langle y-x, t\rangle-y_{0} t\right)^{-2}, x \in \mathbb{R}^{2}$ may be expressed as $f_{t}(x)=\Phi(\langle x, t\rangle), x \in \mathbb{R}^{2}$, with

$$
\Phi(z)=\left(\langle y, t\rangle-z+y_{0} t\right)\left((\langle y, t\rangle-z)^{2}+y_{0}^{2}\right)^{-1} .
$$

The function $\Phi$ is analytic at all points $z \in \mathbb{C}$ for which $(\langle y, t\rangle-z)^{2}+y_{0}^{2} \neq 0$, so it is analytic in the open strip $|\Im z|<\left|y_{0}\right|$ containing $\left\langle\operatorname{supp}\left(\mathscr{W}_{\mathbf{A}}\right), t\right\rangle \subset \mathbb{R}$ for each $t \in \mathbb{T}$. Hence,

$$
\mathscr{W}_{\mathbf{A}}\left(f_{t}\right)=\mathscr{W}_{\mathbf{A}}(\Phi(\langle\cdot, t\rangle))=\left(\langle y I-\mathbf{A}, t\rangle-y_{0} t I\right)^{-2}
$$

for every $t \in \mathbb{T}$.

For all $\omega \in \mathbb{R}^{3}$ with $|\omega|$ sufficiently large, $G_{\omega}(x)$ has an expansion in terms of monogenic polynomials in $x \in \mathbb{R}^{3}$. Replacing the monogenic polynomials with symmetrised products of the operators $A_{1}$ and $A_{2}$, we obtain $G_{\omega}(\mathbf{A})=\mathscr{W}_{\mathbf{A}}\left(G_{\omega}\right)$ for all $\omega$ outside a sufficiently large ball in $\mathbb{R}^{3}$ [17, Equation (5)], [18, Equation (5)]. This is the analogue of the Neumann series for $(\lambda I-A)^{-1},|\lambda|>\|A\|$, in the case that $\mathbf{A}$ is a single operator $A$. The right hand side of (9) has the same representation for all $\omega$ outside a sufficiently large ball in $\mathbb{R}^{3}$.

\section{The monogenic spectrum}

Let $\mathbf{A}=\left(A_{1}, A_{2}\right)$ be a pair of hermitian matrices. Where convenient, we shall represent the $(n \times n)$ matrix associated with $\mathbf{A}$ as $A=A_{1}+i A_{2}$ in order to avoid hats and tildes. In the same spirit, $\mathbb{C}$ is identified with $\mathbb{R}^{2}$ and $\mathbb{R}^{2}$ is identified with the subspace $\{0\} \times \mathbb{R}^{2}$ of $\mathbb{R}^{3}$. We adopt the convention that a point $\omega \in \mathbb{R}^{3}$ is written as $y+y_{0} e_{0}$ for $y \in \mathbb{R}^{2}$ and $y_{0} \in \mathbb{R}$. For a $d$-tuple $B=\left(B_{1}, \ldots, B_{d}\right)$ of $(n \times n)$ matrices and $\xi \in \mathbb{C}^{d}$, the notation $\langle B, \xi\rangle$ is used to denote the matrix $\sum_{j=1}^{d} B_{j} \xi_{j}$.

We are concerned with the compact set $\gamma(\mathbf{A}) \subset \mathbb{R}^{2}$ of points at which the Cauchy kernel $\omega \mapsto G_{\omega}(\mathbf{A})$ has a discontinuity as $\omega \in \mathbb{R}^{3}$ approaches the subspace $\{0\} \times \mathbb{R}^{2}$ of $\mathbb{R}^{3}$ from above $\left(\omega_{0} \rightarrow 0^{+}\right)$and below $\left(\omega_{0} \rightarrow 0^{-}\right)$.

To this end, we examine the integral (9) more closely. Let $y=\left(y_{1}, y_{2}\right) \in \mathbb{R}^{2}$. We interpret $\mathbf{B}(y)=y I-\mathbf{A}$ as the pair $\left(B_{1}(y), B_{2}(y)\right)$ of matrices with $B_{j}(y)=y_{j} I-A_{j}$ for $j=1,2$. Then, appealing to the identity $t^{2}=-1$ for $t \in \mathbb{T} \subset \mathbb{R}_{(2)}$ with respect to 
multiplication in the Clifford algebra, for $y_{0} \neq 0$ the integrand of (9) can be written down explicitly as

$$
\begin{aligned}
\left(\langle\mathbf{B}(y), t\rangle-y_{0} t I\right)^{-2}= & \left(\langle\mathbf{B}(y), t\rangle+y_{0} t I\right)^{2}\left(\langle\mathbf{B}(y), t\rangle^{2}+y_{0}^{2} I\right)^{-2} \\
= & \left(\langle\mathbf{B}(y), t\rangle^{2}-y_{0}^{2} I\right)\left(\langle\mathbf{B}(y), t\rangle^{2}+y_{0}^{2} I\right)^{-2} \\
& +2 y_{0} t\langle\mathbf{B}(y), t\rangle\left(\langle\mathbf{B}(y), t\rangle^{2}+y_{0}^{2} I\right)^{-2}
\end{aligned}
$$

The points $t \in \mathbb{T}$, where $\langle\mathbf{B}(y), t\rangle$ is not invertible, will dominate the integral (9) as $y_{0} \rightarrow 0^{+}$and $y_{0} \rightarrow 0^{-}$, respectively. This suggests to investigate the zeros of

$$
\operatorname{det}\langle\mathbf{B}(y), t\rangle=\operatorname{det}\left(B_{1}(y) t_{1}+B_{2}(y) t_{2}\right) .
$$

Now suppose that $t=\left(t_{1}, t_{2}\right)=(\cos \theta, \sin \theta)$ for $-\pi<\theta<\pi$ and let $z=e^{i \theta}$. Then $t_{1}=\left(z+z^{-1}\right) / 2$ and $t_{2}=\left(z-z^{-1}\right) / 2 i$, so that

$$
\begin{aligned}
\operatorname{det}\langle\mathbf{B}(y), t\rangle= & (2 z)^{-n} \operatorname{det}\left(B_{1}(y)\left(z^{2}+1\right)-i B_{2}(y)\left(z^{2}-1\right)\right) \\
= & (2 z)^{-n} \operatorname{det}\left(\left(B_{1}(y)-i B_{2}(y)\right) z^{2}+\left(B_{1}(y)+i B_{2}(y)\right)\right) \\
= & (2 z)^{-n} \operatorname{det}\left(B_{1}(y)-i B_{2}(y)\right) \\
& \times \operatorname{det}\left(z^{2} I+\left(B_{1}(y)-i B_{2}(y)\right)^{-1}\left(B_{1}(y)+i B_{2}(y)\right)\right)
\end{aligned}
$$

if $B_{1}(y)-i B_{2}(y)$ is invertible.

Fix $y \in \mathbb{R}^{2}$ and let $T=B_{1}(y)+i B_{2}(y)$. Then in the case that $T$ and hence, $T^{*}$, is invertible, the set of points $t \in \mathbb{T}$ where $\operatorname{det}\langle\mathbf{B}(y), t\rangle=0$ is in two-to-one correspondence with $\left[\sigma\left(-\left(T^{*}\right)^{-1} T\right)\right] \cap \mathbb{\pi}$ : if $\zeta$ is an element of the set $\left[\sigma\left(-\left(T^{*}\right)^{-1} T\right)\right] \cap \mathbb{T}$, then the corresponding $t \in \mathbb{T}$ is $\pm \zeta^{1 / 2}$.

For $t \in \mathbb{T}$, the equation $\operatorname{det}\langle\mathbf{B}(y), t\rangle=\operatorname{det}\langle y I-\mathbf{A}, t\rangle=0$ has a geometric interpretation. Let $t^{\perp} \in \mathbb{T}$ be orthogonal to $t$ in $\mathbb{R}^{2}$. Then the line

$$
L_{y, t}=\left\{y+\lambda t^{\perp} \mid \lambda \in \mathbb{R}\right\}
$$

passes through $y \in \mathbb{R}^{2}$ and has the property that $\langle x, t\rangle \in \sigma(\langle\mathbf{A}, t\rangle)$ for all $x \in L_{y, t}$.

As we will see later, the number of such lines that exist for a point $y$ and for all points in a neighbourhood of $y$, is decisive for whether the point $y$ belongs to $\operatorname{supp}\left(\mathscr{W}_{\mathrm{A}}\right)$. We introduce the following definition to isolate those points $y \in \mathbb{R}^{2}$ for which this is the maximum number possible.

DEFINITION 6.1. Let $A$ be a $(n \times n)$ matrix and let $\mathbf{R}(A)$ be the set of all points $\lambda \in \rho(A)$ such that in some neighbourhood $U \subset \rho(A)$ of $\lambda$ in $\mathbb{C}$,

$$
\sigma\left(\left((\zeta I-A)^{*}\right)^{-1}(\zeta I-A)\right) \subset \mathbb{T}
$$

for each $\zeta \in U$. 
The set $\mathbf{R}(A)$ is necessarily an open set. If the matrices $A_{1}$ and $A_{2}$ commute, that is. if $A=A_{1}+i A_{2}$ is a normal matrix, then the set $\mathbf{R}(A)$ is readily described. In this case $\left((\zeta I-A)^{*}\right)^{-1}(\zeta I-A)$ is a unitary matrix for all $\zeta \in \rho(A)$, so that $\mathbf{R}(A)=\rho(A)$.

Condition (12) may be restated by saying that $\mathbf{R}(A)$ is the set of all $\lambda \in \mathbb{C}$ such that in some neighbourhood $U$ of $\lambda$ in $\mathbb{C}$, every solution $z \in \mathbb{C}$ of the equation

$$
\operatorname{det}\left((\zeta I-A)^{*} z+(\zeta I-A)\right)=0
$$

with $\zeta \in U$ satisfies $|z|=1$.

If $\zeta \in \sigma(A)$, then $z=0$ is a solution of (13)-such points are excluded. Note that. in the notation above, this covers the case where $B_{1}(y)-i B_{2}(y)$ is not invertible. For $y \in \rho(A)$, we have

$$
\operatorname{det}\left((y I-A)^{*} z+(y I-A)\right)=\overline{\operatorname{det}(y I-A)} \operatorname{det}\left(z I+\left((y I-A)^{*}\right)^{-1}(y I-A)\right) .
$$

Hence $\operatorname{det}\left((y I-A)^{*} z+(y I-A)\right)$ is a polynomial of degree $n$ in $z$ and there are $n$ solutions $z \in \mathbb{C}$ of (13) counting multiplicity. To each $z \in \mathbb{T}$, there corresponds a line $L_{y, z^{1 / 2}}$ in $\mathbb{R}^{2}$. If all the solutions $z \in \mathbb{C}$ satisfy $|z|=1$, that is, if $y \in \mathbf{R}(A)$. then this says that the number of lines $L_{y, t}, t \in \mathbb{T}$, passing through $y \in \mathbb{R}^{2} \equiv \mathbb{C}$, is the maximum possible. In particular, counting multiplicity, the maximum number of lines $L_{y, t}, t \in \mathbb{T}$, that can possibly pass through $y$ is $n$.

The following simple condition guarantees that a point $y \in \mathbb{R}^{2}$ belongs to $\mathbf{R}(A)$. Let $W(A)$ denote the numerical range $\left\{\langle A v, v\rangle \mid v \in \mathbb{C}^{n},\|v\|=1\right\}$ of the $(n \times n)$ matrix $A=A_{1}+i A_{2}$.

Proposition 6.2. Let $A$ be $a(n \times n)$ matrix. Then $\mathbb{C} \backslash W(A) \subseteq \mathbf{R}(A)$. Consequently, $\mathbb{C} \backslash \mathbf{R}(A)$ is a nonempty compact subset of the numerical range $W(A)$ of the matrix $A$.

Proof. Firstly, $\sigma(A) \subset W(A)$, so if $\lambda \in \mathbb{C}$ lies outside $W(A)$ then $\lambda \in \rho(A)$. Moreover, for every $z \in \mathbb{C}$, the inclusion

$$
\sigma\left((\lambda I-A)^{*} z+(\lambda I-A)\right) \subseteq W\left((\lambda I-A)^{*} z+(\lambda I-A)\right)
$$

holds. Hence, for any complex number $z$ for which $0 \in \sigma\left((\lambda I-A)^{*} z+(\lambda I-A)\right)$, there exists $v \in \mathbb{C}^{n}$ with $|v|=1$ such that $\left\langle(\lambda I-A)^{*} z v, v\right\rangle+\langle(\lambda I-A) v, v\rangle=0$. Here $\langle\cdot, \cdot\rangle$ is the inner product of $\mathbb{C}^{n}$. Because $\lambda-\langle A u, u\rangle \neq 0$ for all $u \in \mathbb{C}^{n}$ with $|u|=1$, the complex number

$$
z=-\frac{\lambda-\langle A v, v\rangle}{\bar{\lambda}-\overline{\langle A v, v\rangle}}
$$

has modulus one. Consequently, $\lambda \in \mathbf{R}(A)$. 
REMARK 6.3. The same proof works with the analogous definition of $\mathbf{R}(A)$ if $A$ is a bounded linear operator on a Hilbert space. If $A$ is normal, then $\mathbf{R}(A)=\rho(A)$ and $\mathscr{W}_{\mathrm{A}}$ is the spectral measure of $A$ supported on $\sigma(A)$.

Our aim is to prove the following result strengthening Proposition 6.2 and providing a geometric characterisation of the support $\operatorname{supp}\left(\mathscr{W}_{\mathbf{A}}\right)$ of the Weyl functional calculus and for the monogenic spectrum $\gamma(\mathbf{A})$ of a pair of hermitian matrices.

THEOREM 6.4. Let $\mathbf{A}=\left(A_{1}, A_{2}\right)$ be a pair of hermitian matrices and $A=A_{1}+i A_{2}$. Then the equalities $\operatorname{supp}\left(\mathscr{W}_{\mathbf{A}}\right)=\gamma(\mathbf{A})=\mathbb{R}^{2} \backslash \mathbf{R}(A)$ hold.

The equality $\operatorname{supp}\left(\mathscr{W}_{\mathbf{A}}\right)=\gamma(\mathbf{A})$ is proved in [17] for any $d$-tuple $\mathbf{A}$ of bounded selfadjoint operators, so this work is concerned with the second equality for hermitian matrices $A_{1}, A_{2}$-the geometric characterisation of $\gamma(\mathbf{A})$.

The spectrum $\sigma(A)$ of the matrix $A=A_{1}+i A_{2}$ is clearly contained in the numerical range $W(A)=\operatorname{co}\left(\operatorname{supp}\left(\mathscr{W}_{\mathrm{A}}\right)\right)$ of $A$. The following immediate consequence of Theorem 6.4 and the fact that $\mathbf{R}(A) \subseteq \rho(A)$ strengthens this observation.

COROllaRY 6.5. Let $\mathbf{A}=\left(A_{1}, A_{2}\right)$ be a pair of hermitian matrices and $A=$ $A_{1}+i A_{2}$. Then $\sigma(A) \subseteq \operatorname{supp}\left(\mathscr{W}_{\mathrm{A}}\right)$.

A bounded linear operator on a Hilbert space is called normal if it commutes with its adjoint. The following consequence of Theorem 6.4 characterises the situation in which the inclusion in Corollary 6.5 is an equality.

COROLlaRY 6.6. Let $\mathbf{A}=\left(A_{1}, A_{2}\right)$ be a pair of hermitian matrices. Set $A=$ $A_{1}+i A_{2}$. The following conditions are equivalent:

(i) $A$ is a normal matrix.

(ii) $\operatorname{supp}\left(\mathscr{W}_{\mathbf{A}}\right)$ has empty interior.

(iii) $\sigma\left(\left((\lambda I-A)^{*}\right)^{-1}(\lambda I-A)\right) \subset \mathbb{T}$ for all $\lambda \in \rho(A)$.

(iv) $\sigma(A)=\operatorname{supp}\left(\mathscr{W}_{\mathbf{A}}\right)$.

Proof. If $A$ is a normal matrix, then the distribution $\mathscr{W}_{\mathrm{A}}$ is associated with the spectral measure of $A$ supported by the finite set of joint eigenvalues of $A$, so the implication (i) $\Rightarrow$ (ii) is immediate. The definition of the set $\mathbf{R}(A)$ and Theorem 6.4 shows that (iv) follows from (iii). The implication (iv) $\Rightarrow$ (i) is proved in $[12,29]$, so it remains to establish (ii) $\Rightarrow$ (iii).

Suppose that the negation of (iii) holds and that $\lambda \in \rho(A)$ has an eigenvalue of the matrix $\left((\lambda I-A)^{*}\right)^{-1}(\lambda I-A)$ lying outside the unit circle $\mathbb{T}$. Then the same holds in a neighbourhood of $\lambda$ because the unordered $n$-tuple of eigenvalues of the matrix valued function $\lambda \mapsto\left((\lambda I-A)^{*}\right)^{-1}(\lambda I-A)$ depends continuously on the parameter $\lambda[22$, 
Theorem II.5.1]. Hence, $\mathbb{C} \backslash \mathbf{R}(A)$ has nonempty interior. According to Theorem 6.4, $\operatorname{supp}\left(\mathscr{W}_{\mathbf{A}}\right)$ has nonempty interior.

\section{The numerical range of matrices}

Let $\mathbf{A}=\left(A_{1}, A_{2}\right)$ be a pair of $(n \times n)$ hermitian matrices. Set $A=A_{1}+i A_{2}$. An application of the Paley-Wiener Theorem yields that the convex hull of the support $\operatorname{supp}\left(\mathscr{W}_{\mathrm{A}}\right)$ of the associated Weyl distribution $\mathscr{W}_{\mathrm{A}}$ coincides with the numerical range $W(A)=\left\{\langle A x, x\rangle \mid x \in \mathbb{C}^{n},\|x\|=1\right\}$ of the matrix $A$. For more precise information on the location of $\operatorname{supp}\left(\mathscr{W}_{\mathbf{A}}\right)$ within the numerical range of $A$, we need to have a closer look at the fine structure of $W(A)$.

Of particular interest are certain plane algebraic curves associated with $A$ that were first investigated by $\mathrm{R}$. Kippenhahn in 1952 . We briefly recall the concepts involved.

Let $\mathbb{F}=\mathbb{R}$ or $\mathbb{C}$. For $0 \leq k \leq 3$, the Grassmannian $G_{3, k} \mathbb{F}$, defined as the set of all $k$-dimensional $\mathbb{F}$-subspaces of $\mathbb{F}^{3}$, is a compact analytic $\mathbb{F}$-manifold of dimension $k(3-k)$. It has a natural topology, induced by the differential structure of the manifold, which is determined, for example, by the metric $h$ on $G_{3, k} \mathbb{F}$ with

$$
h(U, V)=\sup _{v \in V,|v|=1} \inf _{u \in U,|u|=1}\|u-v\| \quad \text { for all } U, V \in G_{3, k} \mathbb{F} .
$$

The projective plane $\mathrm{PG}\left(\mathbb{F}^{3}\right)$ over $\mathbb{F}$ is given by $\mathrm{PG}\left(\mathbb{F}^{3}\right)=\bigcup_{0 \leq k \leq 3} G_{3, k} \mathbb{F}$. The 1and 2-dimensional subspaces of $\mathbb{F}^{3}$ are usually called the points and lines in $\mathrm{PG}\left(\mathbb{F}^{3}\right)$, respectively.

By a common abuse of notation we introduce homogeneous coordinates for the points in $\operatorname{PG}\left(\mathbb{F}^{3}\right)$ as $\left(u_{1}: u_{2}: u_{3}\right)=\mathbb{F}\left(u_{1}, u_{2}, u_{3}\right)$. The coordinates of a vector in $\mathbb{F}^{3}$ are expressed with respect to the standard basis for $\mathbb{F}^{3}$.

A polarity of $\operatorname{PG}\left(\mathbb{F}^{3}\right)$ is a bijection on $\mathrm{PG}\left(\mathbb{F}^{3}\right)$ which reverses the inclusion of subspaces and the square of which equals the identity mapping. The standard polarity $\pi$ is characterised by $u^{\pi}=\left\{v \in \mathbb{F}^{3} \mid \sum_{j=1}^{3} u_{j} v_{j}=0\right\}$ for all $u \in G_{3,1} \mathbb{F}$, which gives $u^{\pi} \in G_{3,2} \mathbb{F}$. Using the polarity $\pi$, we can also introduce homogeneous coordinates for the lines in $\operatorname{PG}\left(\mathbb{F}^{3}\right)$ by setting $\left[v_{1}: v_{2}: v_{3}\right]=\left(v_{1}: v_{2}: v_{3}\right)^{\pi}$.

A nonempty subset $C$ of $G_{3,1} \mathbb{F}$ is called a plane $\mathbb{F}$-algebraic curve if it is the zero locus of a homogeneous 3-variate polynomial over $\mathbb{F}$. The defining polynomial of $C$ is not uniquely determined: if $f$ defines the curve, then so does, for example, $f^{k}$ for any $k \geq 1$. However, every curve $C$ has a defining polynomial of minimal degree which is unique up to a constant factor. A curve is said to be irreducible if it has an irreducible defining polynomial. Since a polynomial ring over a field is a unique factorisation domain, each algebraic curve $C$ is the union of finitely many irreducible curves. If $C_{1}, \ldots, C_{k}$ are the irreducible components of $C$ with irreducible defining 
polynomials $f_{1}, \ldots, f_{k}$, then $f=f_{1} \cdots f_{k}$ is a defining polynomial of $C$ of minimal degree. We call $f$ a minimal polynomial of $C$. Note that an irreducible real algebraic curve is not necessarily connected.

Let $f$ be a minimal polynomial of the algebraic curve $C=\left\{u \in G_{3,1} \mathbb{F} \mid f(u)=0\right\}$. A point $u \in C$ is called singular or a singularity of $C$ if $\left(\partial f / \partial u_{j}\right)(u)=0$ for $j=1,2,3$. Observe that $C$ has at most finitely many singular points. These are the singular points of the irreducible components of $C$ together with the points of intersection of any two of these components. A nonsingular point $u \in C$ is called a simple point of $C$. The curve $C$ is the topological closure of its simple points. Also, to every simple point $u \in C$, there exists a neighbourhood of $u$ in which $C$ admits a smooth parametrization.

Let $C$ be an irreducible plane algebraic curve with minimal polynomial $f$. At each simple point $u \in C$, we have a unique tangent line to $C$ which is given by

$$
\mathscr{T}_{u} C=\left[\frac{\partial f}{\partial u_{1}}(u): \frac{\partial f}{\partial u_{2}}(u): \frac{\partial f}{\partial u_{3}}(u)\right] .
$$

If $C$ is not a projective line or a point, then it is well-known that the set $\left\{\left(\mathscr{T}_{u} C\right)^{\pi} \mid u \in\right.$ $C$ simple\} is contained in a unique irreducible algebraic curve $C^{*}$, the so-called dual curve of $C$. In fact, since an algebraic curve has at most finitely many singularities, the dual curve is the topological closure of the set $\left\{\left(\mathscr{T}_{u} C\right)^{\pi} \mid u \in C\right.$ simple $\}$. We have $C^{* *}=C$. If $C$ is a projective line, then $\left\{\left(\mathscr{T}_{u} C\right)^{\pi} \mid u \in C\right\}$ consists of a single point $u$ in PG $\left(\mathbb{F}^{3}\right)$. In this case, we set $C^{*}=\{u\}$ and define $C^{* *}$ to be the image under $\pi$ of the set of all lines in $\mathrm{PG}\left(\mathbb{F}^{3}\right)$ which pass through $u$. This again yields $C^{* *}=C$. The dual curve of a general plane algebraic curve $C$ is the union of the dual curves of its irreducible components. In particular, $C$ and $C^{*}$ have the same number of irreducible components.

In general, it is difficult to derive an explicit equation for the dual curve $C^{*}$ from the given equation of a curve $C$. However, from the above we obtain the following criterion for a point in $\mathrm{PG}\left(\mathbb{F}^{3}\right)$ to belong to $C^{*}$.

LEMMA 7.1. Let $\left(x_{1}: x_{2}: 1\right) \in G_{3,1} \mathbb{F}$. If there exists a smooth local parametrization $\zeta \mapsto(c(\zeta): s(\zeta): \mu(\zeta))$ of $C$, for $\zeta$ in an open set $U \subseteq \mathbb{F}$, and a point $z \in U$ such that $x_{1} c(z)+x_{2} s(z)+\mu(z)=0$ and $x_{1} c^{\prime}(z)+x_{2} s^{\prime}(z)+\mu^{\prime}(z)=0$, then the point $\left(x_{1}: x_{2}: 1\right)$ belongs to $C^{*}$.

ProOF. The two points $(c(z): s(z): \mu(z))$ and $\left(c^{\prime}(z): s^{\prime}(z): \mu^{\prime}(z)\right)$ span the tangent line $\mathscr{T}_{(c(z): s(z): \mu(z))} C$ to $C$ at $(c(z): s(z): \mu(z))$. The equations

$$
x_{1} c(z)+x_{2} s(z)+\mu(z)=0 \quad \text { and } \quad x_{1} c^{\prime}(z)+x_{2} s^{\prime}(z)+\mu^{\prime}(z)=0
$$

imply that $\left(x_{1}: x_{2}: 1\right)=\left(\mathscr{T}_{(c(z): s(z): \mu(z))} C\right)^{\pi}$. Hence $\left(x_{1}: x_{2}: 1\right)$ belongs to the dual curve $C^{*}$ of $C$. 
The details and further information on complex algebraic curves can be found, for example, in [31]. The literature for the real case is somewhat less easy to access. As a general reference to the theory of real algebraic geometry, see [8].

Let $A=A_{1}+i A_{2} \in \mathscr{L}\left(\mathbb{C}^{n}\right)$. Following Kippenhahn [23], we define the complex algebraic curve $C_{\mathrm{C}}(A)$ in the complex projective plane $\mathrm{PG}\left(\mathbb{C}^{3}\right)$ by setting its dual curve to be

$$
D_{\mathrm{C}}(A)=\left\{(c: d: \mu) \in G_{3,1} \mathbb{C} \mid \operatorname{det}\left(c A_{1}+d A_{2}+\mu I\right)=0\right\} .
$$

In [23], Kippenhahn showed that the real part $C_{\mathbb{R}}(A)$ of the curve $C_{\mathbf{C}}(A)=D_{\mathbf{C}}(A)^{*}$ is contained in the affine subplane $F=\left\{\left(\alpha_{1}: \alpha_{2}: 1\right) \mid\left(\alpha_{1}, \alpha_{2}\right) \in \mathbb{R}^{2}\right\}$ of $\operatorname{PG}\left(\mathbb{R}^{3}\right)$ and, identifying $F$ with $\mathbb{R}^{2}$ in the canonical way, that the convex hull $\operatorname{co}\left(C_{\mathbb{R}}(A)\right)$ of $C_{\mathbb{R}}(A)$ is precisely the numerical range of $A$.

The curve $C_{\mathbb{R}}(A)$ considered as a real algebraic curve in $\mathrm{PG}\left(\mathbb{R}^{3}\right)$ is the dual curve of the real part of $D_{\mathrm{C}}(A)$ given by

$$
D_{\mathbb{R}}(A)=\left\{(c: d: \mu) \in G_{3,1} \mathbb{R} \mid \operatorname{det}\left(c A_{1}+d A_{2}+\mu I\right)=0\right\} .
$$

Every point $u \in D_{\mathbb{R}}(A)$ has a representation $(\cos \theta: \sin \theta: \mu)$ for some $\theta \in[0, \pi)$ and $\mu \in \mathbb{R}$. As $u$ is a zero of $\operatorname{det}\left(c A_{1}+d A_{2}+\mu I\right)$, it follows that $-\mu$ is an eigenvalue of the operator $\mathscr{A}(\theta)=\cos \theta A_{1}+\sin \theta A_{2}$.

Note that the points in $D_{\mathbb{R}}(A)$ are in one-to-one correspondence with the lines $L_{y, t}$ in $\mathbb{R}^{2}$ defined in (11), satisfying $\langle x, t\rangle \in \sigma(\langle\mathbf{A}, t\rangle)$ for all $x \in L_{y, t}$. For $u=(\cos \theta$ : $\sin \theta: \mu) \in D_{\mathbb{R}}(A)$, take $t=(\cos \theta, \sin \theta) \in \mathbb{T}$ and $y \in \mathbb{R}^{2}$ such that $\langle y, t\rangle=-\mu$. Then $u^{\pi}$ is the two dimensional subspace $\bigcup\left\{\left(x_{1}: x_{2}: 1\right) \mid\left(x_{1}, x_{2}\right) \in L_{y, t}\right\}$ of $\mathbb{R}^{3}$, that is, $L_{y, t} \times\{1\}$ is the line in which the plane $u^{\pi}$ normal to $u$ in $\mathbb{R}^{3}$ cuts the plane $\left\{x_{3}=1\right\}$.

With the following result due to Rellich [28], [22, Theorem 6.1, page 120], we obtain local parametrizations of the curve $D_{\mathbb{R}}(A)$. Let $S\left(\mathbb{C}^{n}\right)=\left\{x \in \mathbb{C}^{n}|| x \mid=1\right\}$ be the unit sphere in $\mathbb{C}^{n}$.

LEMMA 7.2 (see $\left[28\right.$, Satz 1]). Let the map $\mathscr{A}: \mathbb{R} \rightarrow \mathscr{L}\left(\mathbb{C}^{n}\right)$ be given by $\mathscr{A}(\theta)=$ $\cos \theta A_{1}+\sin \theta A_{2}$ for $\theta \in \mathbb{R}$. Let $\theta_{0} \in \mathbb{R}$ and $\mu_{0}$ be an eigenvalue with multiplicity $r$ of the operator $\mathscr{A}\left(\theta_{0}\right)$. Then there exists a neighborhood $U$ of $\theta_{0}$ and regular analytic functions $\mu_{j}: U \rightarrow \mathbb{R}$ and $x_{j}: U \rightarrow S\left(\mathbb{C}^{n}\right)$ with $1 \leq j \leq r$, such that $\mu_{j}\left(\theta_{0}\right)=\mu_{0}$, $\mathscr{A}(\theta) x_{j}(\theta)=\mu_{j}(\theta) x_{j}(\theta)$ and $\left\langle x_{j}(\theta), x_{k}(\theta)\right\rangle=\delta_{j k}$ for every $\theta \in U$ and $1 \leq j, k \leq r$.

Given a point $u_{0}=\left(\cos \theta_{0}: \sin \theta_{0}:-\mu_{0}\right) \in D_{\mathbb{R}}(A)$, any of the maps $\theta \mapsto(\cos \theta$ : $\sin \theta:-\mu(\theta)$ ) with $\theta$ in the neighbourhood $U$ of $\theta_{0}$ as given by Lemma 7.2, is then a smooth local parametrization of a component of $D_{\mathbb{R}}(A)$ in a neighbourhood of $u_{0}$. With Lemma 7.1, this yields immediately a complete characterisation of the curve $C_{\mathbb{R}}(A)$. 
LEMMA 7.3. A point $\left(x_{1}: x_{2}: 1\right) \in G_{3,1} \mathbb{F}$ belongs to the curve $C_{\mathbb{R}}(A)$ if and only if there exists a point $u_{0}=\left(\cos \theta_{0}: \sin \theta_{0}:-\mu_{0}\right) \in D_{\mathbb{R}}(A)$, and a local parametrization $\theta \mapsto(\cos \theta: \sin \theta:-\mu(\theta))$ of a component of $D_{\mathbb{R}}(A)$ in a neighbourhood $U$ of $u_{0}$ such that $x_{1} \cos \theta_{0}+x_{2} \sin \theta_{0}-\mu\left(\theta_{0}\right)=0$ and $-x_{1} \sin \theta_{0}+x_{2} \cos \theta_{0}-\mu^{\prime}\left(\theta_{0}\right)=0$. Then

$$
\left(x_{1}, x_{2}\right)=\mu\left(\theta_{0}\right)\left(\cos \theta_{0}, \sin \theta_{0}\right)+\mu^{\prime}\left(\theta_{0}\right)\left(-\sin \theta_{0}, \cos \theta_{0}\right) .
$$

The line $L_{y, t}$ associated with $u_{0}^{\pi}$, as described above, is therefore tangential to the image of $C_{\mathbb{R}}(A)$ in $\mathbb{R}^{2}$ at $\left(x_{1}, x_{2}\right)$ except in the case that $\mu(\theta)=a_{1} \cos \theta+a_{2} \sin \theta$ in a neighbourhood $U$ of $\theta_{0}$. Then the set $\left\{(\cos \theta: \sin \theta: \mu(\theta))^{\pi} \mid \theta \in U\right\}$ corresponds to a family of lines passing through the point $\left(x_{1}, x_{2}\right)=\left(a_{1}, a_{2}\right)$.

LEMMA 7.4. With the exception of a finite set of points in $C_{\mathbb{R}}(A)$, if $\left(x_{1}: x_{2}: 1\right) \in$ $G_{3,1} \mathbb{F}$ belongs to $C_{\mathbb{R}}(A)$, and $u_{0}=\left(\cos \theta_{0}: \sin \theta_{0}: \mu_{0}\right)$ is one of the corresponding points in $D_{\mathbb{R}}(A)$ and $\theta \mapsto(\cos \theta: \sin \theta:-\mu(\theta)), \theta \in U$, is one of the corresponding local parametrizations of a component of $D_{\mathbb{R}}(A)$ in a neighbourhood of $u_{0}$ as given by Lemma 7.3 , then the equation

$$
\left(x_{1}-t \sin \theta_{0}\right) \cos \theta+\left(x_{2}+t \cos \theta_{0}\right) \sin \theta-\mu(\theta)=0
$$

has two real solutions $\theta \in U$ for either small positive $t$ or small negative $t$ and none in $U$ for $t$ of the opposite sign.

PROOF. By Lemma 7.3, the image of the curve $C_{\mathbb{R}}(A)$ in $\mathbb{R}^{2}$ has the local parametrization $\left(x_{1}(\theta), x_{2}(\theta)\right)=\mu(\theta)(\cos \theta, \sin \theta)+\mu^{\prime}(\theta)(-\sin \theta, \cos \theta)$ with $\theta \in U$. Hence, its signed curvature at $\left(x_{1}, x_{2}\right)$ is given by $\left|\mu\left(\phi_{0}\right)+\mu^{\prime \prime}\left(\phi_{0}\right)\right|^{-1}$ (see, for example, [7, formula (3.9)]). So if $\mu\left(\phi_{0}\right)+\mu^{\prime \prime}\left(\phi_{0}\right) \neq 0$, then the image of $C_{\mathbb{R}}(A)$ in $\mathbb{R}^{2}$ is a smooth curve with nonzero curvature in a neighbourhood of $\left(x_{1}, x_{2}\right)$. Hence, there are two tangents with points of tangency in $U$ on one side of the curve and none on the other for $|t|>0$ small enough. The solutions $\theta \in U$ of (15) correspond to the directions of the normals to the tangents.

The points of $C_{\mathbb{R}}(A)$ that we have to exclude correspond to the ones at which the image of $C_{\mathbb{R}}(A)$ in $\mathbb{R}^{2}$ has infinite curvature. Unless $\mu+\mu^{\prime \prime}$ vanishes identically, there exist at most finitely many solutions $\theta$ of $\mu(\theta)+\mu^{\prime \prime}(\theta)=0$ in any compact interval. If $\left(x_{1}: x_{2}: 1\right) \in C_{\mathbb{R}}(A)$ is a point for which the analytic function $\mu+\mu^{\prime \prime}$ vanishes in a neighbourhood $U$ of $\theta_{0}$ in $\mathbb{C}$, there exists $\left(a_{1}, a_{2}\right) \in \mathbb{R}^{2}$ such that $\mu(\theta)=a_{1} \cos \theta+a_{2} \sin \theta$ for all $\theta \in U$. However, inspection of (14) shows that then $x=\left(x_{1}, x_{2}\right)=\left(a_{1}, a_{2}\right)$ is a point of $C_{\mathbb{R}}(A)$ through which the family of lines $L_{x, t}$, $t \in \mathbb{T}$, passes. In particular, $x$ belongs to the finite set $\sigma\left(A_{1}\right) \times \sigma\left(A_{2}\right)$. 
Local coordinates. Define the function $s: \mathbb{C} \backslash\{0\} \rightarrow \mathbb{C}^{2}$ by

$$
s(z)=\left(\frac{1}{2}(z+1 / z), \frac{1}{2 i}(z-1 / z)\right), \quad \text { for } z \in \mathbb{C} \backslash\{0\} .
$$

From now on, we drop the subscript $\mathbb{R}$ from the Kippenhahn curves $C_{\mathbb{R}}(A)$ and denote them by $C(A)$. Furthermore, we identify $\left(y_{1}: y_{2}: 1\right) \in C_{\mathbb{R}}(A)$ with $y=\left(y_{1}, y_{2}\right) \in$ $\mathbb{R}^{2}$, so that $C(A)$ is a subset of $\mathbb{R}^{2}$.

According to the discussion preceding Definition 6.1 we have the following alternative formulation of the set $\mathbf{R}(A)$.

Proposition 7.5. Let $\mathbf{A}=\left(A_{1}, A_{2}\right)$ be a pair of $(n \times n)$ hermitian matrices. Then $\mathbf{R}(A)$ is the set of all $\lambda \in \mathbb{R}^{2}$ for which there exists a neighbourhood $U$ of $\lambda$ in $\mathbb{R}^{2}$, with the property that for each $x \in U$, every solution $z \in \mathbb{C} \backslash\{0\}$ of the equation

$$
\operatorname{det}(\langle x I-\mathbf{A}, s(z)\rangle)=0
$$

satisfies $|z|=1$.

Let $y \in \mathbb{R}^{2}$ and suppose that $\zeta \in \mathbb{C} \backslash\{0\}$ is a point at which

$$
\operatorname{det}(\langle y I-\mathbf{A}, s(\zeta)\rangle)=0
$$

If $y \in \mathbf{R}(A)$, then $\zeta$ necessarily belongs to $\mathbb{T}$, and the set $Z_{y}$ of all such $\zeta \in \mathbb{T}$ is finite.

Suppose that $\zeta \in \mathbb{T}$. Then $s(\zeta) \in \mathbb{T}$ and the matrix $\langle\mathbf{A}, s(\zeta)\rangle$ is hermitian. By a result of Rellich [22, Theorem II.6.1], there exists a neighbourhood $V_{\zeta}$ of $\zeta$ in $\mathbb{C} \backslash\{0\}$, a positive integer $m \leq n$, analytic $\mathscr{L}\left(\mathbb{C}^{n}\right)$-valued projections $P_{1}(z), \ldots, P_{m}(z)$ with $\sum_{j=1}^{m} P_{j}(z)=I$ and analytic functions $\mu_{1}(z), \ldots, \mu_{m}(z)$ defined for $z \in V_{\zeta}$ such that for each $j=1, \ldots, m$, the equation

$$
\operatorname{det}\left(\mu_{j}(z) I-\langle\mathbf{A}, s(z)\rangle\right)=0, \quad z \in V_{\zeta},
$$

holds, $\langle y, s(\zeta)\rangle-\mu_{1}(\zeta)=0$ and

$$
\langle\mathbf{A}, s(z)\rangle=\sum_{j=1}^{m} \mu_{j}(z) P_{j}(z), \quad z \in V_{\zeta} .
$$

Here $P_{j}(z)$ is the projection onto an eigenspace for the eigenvalue $\mu_{j}(z)$ of $\langle\mathbf{A}, s(z)\rangle$.

Set $\lambda_{j, y}(z)=\langle y, s(z)\rangle-\mu_{j}(z)$ for $j=1, \ldots, m$ and $z \in V_{\zeta}$. Then

$$
\langle y I-\mathbf{A}, s(z)\rangle=\sum_{j=1}^{m} \lambda_{j, y}(z) P_{j}(z), \quad z \in V_{\zeta} .
$$


It turns out that the functions $\mu_{j}$ and projections $P_{j}, j=1, \ldots, m$, can be analytically continued along any arc that avoids a certain finite exceptional set of points [5, Theorem 3.3.12]. Therefore, formula (18) may also be valid in a neighbourhood $V_{\zeta}$ of points $\zeta \in \mathbb{C} \backslash\{0\}$ not on the unit circle $\mathbb{T}$.

It can happen that two of the eigenvalues $\lambda_{j, y}(z)$ and $\lambda_{\ell, y}(z)$ of $\langle y I-\mathbf{A}, s(z)\rangle$ are equal at a particular complex number $z$. In particular, there may exist an integer $1<k \leq m$ such that $\lambda_{j, y}(\zeta)=0$ for all $j=1, \ldots, k$. According to the interpretation preceding Definition 6.1 and the definition of $C(A)$, if $\zeta \in \mathbb{T}$, then there exist $k$ coincident tangent lines from $y$ to $C(A)$ with normal $\zeta$.

LEMMA 7.6. Let $x \in \mathbb{R}^{2}$, let $\zeta \in \mathbb{C} \backslash\{0\}$ be a complex number and $V_{\zeta}$ an open neighbourhood of $\zeta$ in $\mathbb{C}$ for which (18) is an analytic parametrization in $V_{\zeta}$ with $\lambda_{1, x}(\zeta)=0$ and $\lambda_{1, x}^{\prime}(\zeta) \neq 0$. Then there exists a unique $C^{\infty}$-function $\phi: U_{x} \rightarrow \mathbb{C}$ defined in a neighbourhood $U_{x}$ of $(0, x)$ in $\mathbb{R}^{3}$ such that $\phi(0, x)=\zeta$ and $\lambda_{1, y}(\phi(\xi, y))=i \xi$ for all $(\xi, y) \in U_{x}$.

Moreover, for $y$ fixed, the function $\xi \mapsto \phi(\xi, y),(\xi, y) \in U_{x}$ is one-to-one and $\lambda_{1, y}^{\prime}(\phi(\xi, y))$ is nonzero for all $(\xi, y) \in U_{x}$. If $\zeta \in \mathbb{T}$, then $\phi(0, y) \in \mathbb{T}$ for all $(0, y) \in U_{x}$.

Proof. Let $U \subset \mathbb{R}^{4}$ be the set $U=V_{\zeta} \times \mathbb{R}^{2}$ and let $\Phi: U \rightarrow \mathbb{R}^{4}$ be defined by

$$
\Phi(z, y)=\left(\lambda_{1, y}(z), y\right)=\left(\langle y, s(z)\rangle-\mu_{1}(z), y\right)
$$

for all $(z, y) \in U$. Here we identify $\mathbb{C}$ with $\mathbb{R}^{2}$ on the right hand side of the equation. The derivative $\Phi^{\prime}(\zeta, x)$ of the function $\Phi$ on the open subset $U$ of $\mathbb{R}^{4}$, as a function of four real variables, is nonsingular at $(\zeta, x) \in U$ because

$$
\operatorname{det}\left(\Phi^{\prime}(\zeta, x)\right)=\left|\lambda_{1, x}^{\prime}(\zeta)\right|^{2} \neq 0
$$

By the inverse function theorem, there exists an open neighbourhood $W$ of $(0, x)$ in $\mathbb{R}^{4}$, an open neighbourhood $U^{\prime}$ of $(\zeta, x)$ in $\mathbb{R}^{4}$ and a diffeomorphism $f: W \rightarrow U^{\prime}$ such that $\Phi \circ f(\alpha, y)=(\alpha, y)$ for all $(\alpha, y) \in W$. In particular, $\Phi^{\prime}$ is nonsingular on $U^{\prime}$.

Then $\phi(\xi, y) \in \mathbb{C}$ is defined on the set $U_{x}$ of all $\left(\xi, y_{1}, y_{2}\right) \in \mathbb{R}^{3}$ such that $\left(0, \xi, y_{1}, y_{2}\right) \in W$, by $f\left(0, \xi, y_{1}, y_{2}\right)=(\phi(\xi, y), y)$, so that $\lambda_{1, y}(\phi(\xi, y))=i \xi$. Because $f$ is a diffeomorphism, the function $(\xi, y) \mapsto \phi(\xi, y)$ is $C^{\infty}$ on $U_{x}$. Furthermore, $(\phi(\xi, y), y) \in U^{\prime}$, so $\left|\lambda_{1, y}^{\prime}(\phi(\xi, y))\right|^{2}=\operatorname{det}\left(\Phi^{\prime}(\phi(\xi, y), y)\right) \neq 0$ for all $(\xi, y) \in U_{x}$.

Now suppose that $\zeta \in \mathbb{T}$. There exists an open neighbourhood $N_{\zeta}$ of $\zeta$ in $\mathbb{T}$ on which $\lambda_{1, x}$ is defined. Let $\Psi: N_{\zeta} \times \mathbb{R}^{2} \rightarrow \mathbb{R}^{3}$ be defined by

$$
\Psi(s, y)=\left(\lambda_{1, y}(t), y\right)=\left(\langle y, t\rangle-\mu_{1}(t), y\right)
$$


for all $(t, y) \in N_{\zeta} \times \mathbb{R}^{2}$. Then $\Psi(\zeta, x)=(0, x)$ and the derivative

$$
\Psi^{\prime}(\zeta, x): T_{(\zeta, x)}\left(\mathbb{T} \times \mathbb{R}^{2}\right) \rightarrow \mathbb{R}^{3}
$$

of $\Psi$ at $(\zeta, x) \in \mathbb{T} \times \mathbb{R}^{2}$ is nonsingular. Here $T_{(\zeta, x)}\left(\mathbb{T} \times \mathbb{R}^{2}\right)$ is the tangent space of $\mathbb{T} \times \mathbb{R}^{2}$ at $(\zeta, x)$. As above, there exists a diffeomorphism $g$ from an open neighbourhood of $(0, x)$ in $\mathbb{R}^{3}$ onto an open neighbourhood of $(t, x)$ is $\mathbb{J} \times \mathbb{R}^{2}$ such that $\Psi \circ g=$ Id. Because $\Psi=\Phi \mid N_{\zeta} \times \mathbb{R}^{2}$, we must have $g(\alpha, y)=f(\alpha, 0, y)$. Hence

$$
(\phi(0, y), y)=f(0,0, y) \in \mathbb{T} \times \mathbb{R}^{2},
$$

proving that $\phi(0, y) \in \mathbb{T}$.

If $\tilde{\Phi}: V_{\zeta} \times \mathbb{C}^{2} \rightarrow \mathbb{C}^{3}$ is defined by $\tilde{\Phi}(z, \eta)=\left(\sum_{j=1}^{2} \eta_{j} s_{j}(z)-\mu_{1}(z), \eta\right)$ for all $z \in V_{\zeta}$ and $\eta \in \mathbb{C}^{2}$, then a similar argument to that above, but replacing $\Phi$ by $\tilde{\Phi}$ and appealing to the inverse function theorem for analytic functions of several variables, shows that $\phi$ is actually the restriction to $U_{x}$ of a function analytic in an open subset of $\mathbb{C}^{3}$.

According to a rephrasing of Lemma 7.3 in terms of our local coordinates, the Kippenhahn curves $C(A)$ for a matrix $A$ are characterised by the following proposition.

PROPOSITION 7.7. The Kippenhahn curves $C(A)$ consist of all points $y \in \mathbb{R}^{2}$ for which there exists a point $\zeta$ belonging to the unit circle $T$ and a neighbourhood $V_{\zeta}$ of $\zeta$ in $\mathbb{C}$ such that there exists an analytic parametrization (18) on $V_{\zeta}$ for which

$$
\lambda_{1, y}(\zeta)=\lambda_{1, y}^{\prime}(\zeta)=0
$$

Of course, in any such parametrization (18), we are at liberty to choose the indices $j=1, \ldots, m$ for the analytic functions $\lambda_{j, y}: V_{\zeta} \rightarrow \mathbb{C}$. In particular, for any $y \in C(A)$, we can choose a neighbourhood $V_{\zeta}$ of $\zeta$ in $\mathbb{C}$ and indices for which (19) holds for $j=1$.

COROLlaRY 7.8. Let $x \in \mathbb{R}^{2} \backslash C(A)$, let $\zeta \in \mathbb{C} \backslash\{0\}$ be a complex number and $V_{\zeta}$ a neighbourhood of $\zeta$ in $\mathbb{C}$ for which $(18)$ is a parametrization with $\lambda_{1, x}(\zeta)=0$. Then there exists a unique $C^{\infty}$-function $\phi: U_{x} \rightarrow \mathbb{C}$ defined in a neighbourhood $U_{x}$ of $(0, x)$ in $\mathbb{R}^{3}$ such that such that $\phi(0, x)=\zeta$ and $\lambda_{1, y}(\phi(\xi, y))=i \xi$ for all $(\xi, y) \in U_{x}$.

Moreover, for $y$ fixed, the function $\xi \mapsto \phi(\xi, y),(\xi, y) \in U_{x}$ is one-to-one and $\lambda_{1, y}^{\prime}(\xi, y)$ is nonzero for all $(\xi, y) \in U_{x}$. If $\zeta \in \mathbb{T}$, then $z(0, y) \in \mathbb{T}$ for all $(0, y) \in U_{x}$.

Proof. By Proposition 7.7, $\lambda_{1, x}^{\prime}(\zeta) \neq 0$, so Lemma 7.6 is applicable. 
The following result describes the relation between the set $\mathbf{R}(A)$ and the Kippenhahn curves $C(A)$.

\section{COROLlaRY 7.9. $\partial \mathbf{R}(A) \subseteq C(A) \subseteq \mathbf{R}(A)^{c}$.}

PROOF. Let $x \in \overline{\mathbf{R}(A)}$. All solutions $\zeta$ of $\operatorname{det}(\langle x, \zeta\rangle I-\langle\mathbf{A}, \zeta\rangle)=0$ satisfy $|\zeta|=1$ because the set-valued function $y \mapsto \sigma\left(\left((y I-A)^{*}\right)^{-1}(y I-A)\right), y \in \rho(A)$, is continuous in the metric of unordered $n$-tuples [22, Theorem II.5.1] and by definition, $\sigma\left(\left((y I-A)^{*}\right)^{-1}(y I-A)\right) \subset \mathbb{T}$ for all $y \in \mathbf{R}(A)$.

For any such $\zeta \in \mathbb{T}$, there exists an analytic parametrization (18) such that $\lambda_{1, x}(\zeta)=0$. Suppose $\lambda_{1, x}^{\prime}(\zeta) \neq 0$. Then by Lemma 7.6, for all $y$ in an open neighbourhood of $x$, we can find $\phi(0, y) \in \mathbb{T}$ such that $\lambda_{1, y}(\phi(0, y))=0$ and $\lambda_{1, y}^{\prime}(\phi(0, y)) \neq 0$.

It follows that if $\lambda_{1, x}^{\prime}(\zeta) \neq 0$ holds for the parametrizations of all solutions $\zeta$, then there is a neighbourhood $U$ of $x$ such that for every $y \in U$, all nonzero complex solutions $z$ of $\operatorname{det}(\langle y, s(z)\rangle I-\langle\mathbf{A}, s(z)\rangle)=0$ satisfy $|z|=1$. This means that $x \in \mathbf{R}(A)$.

Therefore, for every element $x$ of $\partial \mathbf{R}(A)=\overline{\mathbf{R}(A)} \backslash \mathbf{R}(A)$, there must exist a solution $\zeta$ and an analytic parametrization (18) such that $\lambda_{1, x}(\zeta)=0$ and $\lambda_{1, x}^{\prime}(\zeta)=0$. Proposition 7.7 yields that $x \in C(A)$.

To establish the inclusion $C(A) \subseteq \mathbf{R}(A)^{c}$, suppose that $x \in C(A)$. By Lemma 7.4, except possibly for a finite subset $J$ of $C(A)$, there exists a neighbourhood $U$ of $x$ in $\mathbb{R}^{2}$ in which not every solution $z \in \mathbb{C}$ of $\operatorname{det}(\langle y I-\mathbf{A}, s(z)\rangle)=0$ for $y \in U$, belongs to $\mathbb{T}$. More precisely, for $y$ on one side of $C(A)$, there exist at least two solutions belonging to $\mathbb{T}$-two unit normal vectors to the local tangents to $C(A)$ passing through $y$-and for $y$ on the other side of $C(A)$, two solutions that do not belong to $\mathbb{T}$. Moreover, if $x \in J$, then either $x$ is isolated, or any neighbourhood of $x$ contains a point $y \in C(A) \backslash J$ to which the conclusion above applies. In either case, $x \in \mathbf{R}(A)^{c}$.

By considering the direct sum of suitable matrices, the inclusions of Corollary 7.9 can be made to be proper inclusions.

We informally state alternative characterisations of the Kippenhahn curves $C(A)$ :

- The real part of the curve $D_{\mathrm{C}}(A)^{*}$ dual to $D_{\mathrm{C}}(A)=\left\{(c: d: \mu) \in G_{3,1} \mathbb{C} \mid\right.$ $\operatorname{det}\left(c A_{1}+d A_{2}+\mu I\right)=0$, identifying $\left(\alpha_{1}, \alpha_{2}\right) \in \mathbb{R}^{2}$ with $\left(\alpha_{1}: \alpha_{2}: 1\right) \in G_{3,1} \mathbb{C}$.

- The real algebraic curve dual to $D_{\mathbb{R}}(A)=\left\{(c: d: \mu) \in G_{3,1} \mathbb{R} \mid \operatorname{det}\left(c A_{1}+\right.\right.$ $\left.d A_{2}+\mu I\right)=0$, identifying $\left(\alpha_{1}, \alpha_{2}\right) \in \mathbb{R}^{2}$ with $\left(\alpha_{1}: \alpha_{2}: 1\right) \in G_{3,1} \mathbb{R}$.

- All points $y \in \mathbb{R}^{2}$ for which there exists $\zeta \in \mathbb{T}$ and a neighbourhood $V_{\zeta}$ of $\zeta$ in $\mathbb{C}$ such that there exists an analytic parametrization (18) on $V_{\zeta}$ for which $\lambda_{1, y}(\zeta)=\lambda_{1, y}^{\prime}(\zeta)=0$ [Proposition 7.7]. 
- The envelope of all lines $L_{y, s}$ given by (11) for each $y \in \mathbb{R}^{2}$ and $s \in \mathbb{T}$ such that $\langle y, s\rangle \in \sigma(\langle\mathbf{A}, s\rangle)$.

- The singular values of the numerical range map $n_{A}$ associated with the matrix $A$ (see [14] and [21]), with the possible exception of "double tangents" [21, Theorem 3.5].

\section{Examples}

The Weyl functional calculus $\mathscr{W}_{\mathbf{A}}$ for a pair $\mathbf{A}=\left(A_{1}, A_{2}\right)$ of $(2 \times 2)$ hermitian matrices can be calculated explicitly. The support $\gamma(\mathbf{A})$ of $\mathscr{W}_{\mathbf{A}}$ is either the numerical range $W(A)$ of the matrix $A=A_{1}+i A_{2}$, an elliptical plane region with nonempty interior in the case that $A_{1}, A_{2}$ do not commute with each other, or $\gamma(\mathbf{A})$ consists of a single point $\sigma \in \mathbb{R}^{2}$ if $\mathbf{A}=\sigma I$, or otherwise, two distinct joint eigenvalues $\sigma_{1}, \sigma_{2} \in \mathbb{R}^{2}$. Calculations of this nature follow from [2] and are given explicitly in [19].

The case of a pair $\mathbf{A}$ of noncommuting $(3 \times 3)$ hermitian matrices reveals greater geometric structure. If $\mathbf{A}$ has a joint eigenvalue $\sigma \in \mathbb{R}^{2}$, then $\gamma(\mathbf{A})$ consists of $\sigma$ together with the support of the Weyl functional calculus associated with the pair of reduced $(2 \times 2)$ matrices, possibly consisting of the point $\sigma$ together with a disjoint elliptical region.

In the following diagrams, Figures $1-6$, we plot the lines in $\mathbb{R}^{2}$ corresponding to points $(c: d: \lambda) \in D_{\mathbb{R}}(A)$ after the fashion described in Section 7. The displayed lines are tangent to the algebraic curves $C(A)$ or pass through the isolated points belonging to $C(A)$. Such an isolated point exists in Figure 1, although it is not a joint eigenvalue - the two matrices written under Figure 1 have no nontrivial common invariant subspace. The numerical range $W(A)$ of $A$ and the support $\gamma(\mathbf{A})$ of the Weyl functional calculus are equal to the elliptical region-a convex set. This is also an example where $\partial \gamma(\mathbf{A})=\partial \mathbf{R}(A)$ is a proper subset of $C(A)$, see Corollary 7.9.

There is a joint eigenvalue $(1,0)$ in Figure 2 and an ellipse corresponding to the pair of reduced $(2 \times 2)$ matrices. The numerical range $W(A)$ of $A=A_{1}+i A_{2}$ is the convex hull of the point $(1,0)$ and the ellipse. The support $\gamma(\mathbf{A})$ of the Weyl functional calculus is the union of the elliptical region and the isolated point $(1,0)$. The convex hull of $\gamma(\mathbf{A})$ coincides with $W(A)$, as required by the Paley-Wiener Theorem.

Figures 3-6 display the Kippenhahn curves $C(A)$ associated with various perturbations of the matrices $A_{1}, A_{2}$ in Figure 2 . The boundary of the lightly shaded regions with single lines passing through them are the algebraic curves $C(A)$. The convex hull of $C(A)$ is the numerical range $W(A)$ of $A_{1}+i A_{2}$. Theorem 6.4 says that, in each diagram, the lightly shaded region is actually the support $\gamma(\mathbf{A})$ of the Weyl functional calculus $\mathscr{W}_{\mathbf{A}}$ for $\mathbf{A}$.

By way of comparison with the general theory of $[3,4]$, the support of the funda- 


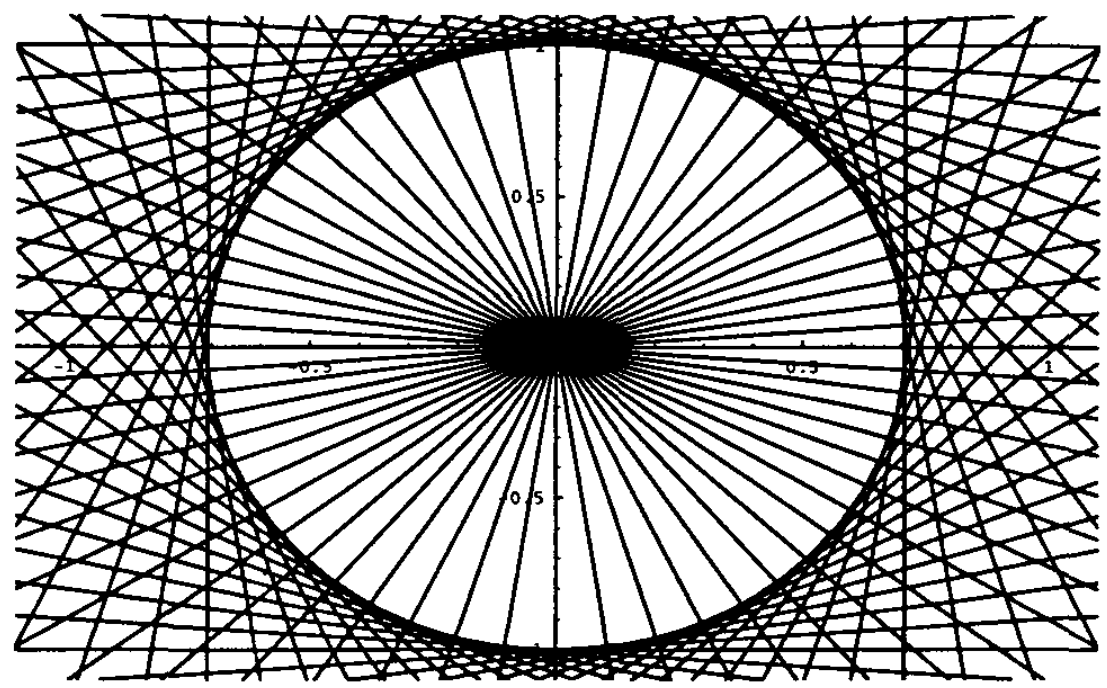

$$
\mathbf{A}=\left(\left(\begin{array}{ccc}
0 & 1 / 2 & 0 \\
1 / 2 & 0 & 1 / 2 \\
0 & 1 / 2 & 0
\end{array}\right),\left(\begin{array}{ccc}
1 & 0 & 0 \\
0 & 0 & 0 \\
0 & 0 & -1
\end{array}\right)\right)
$$

FIGURE 1.

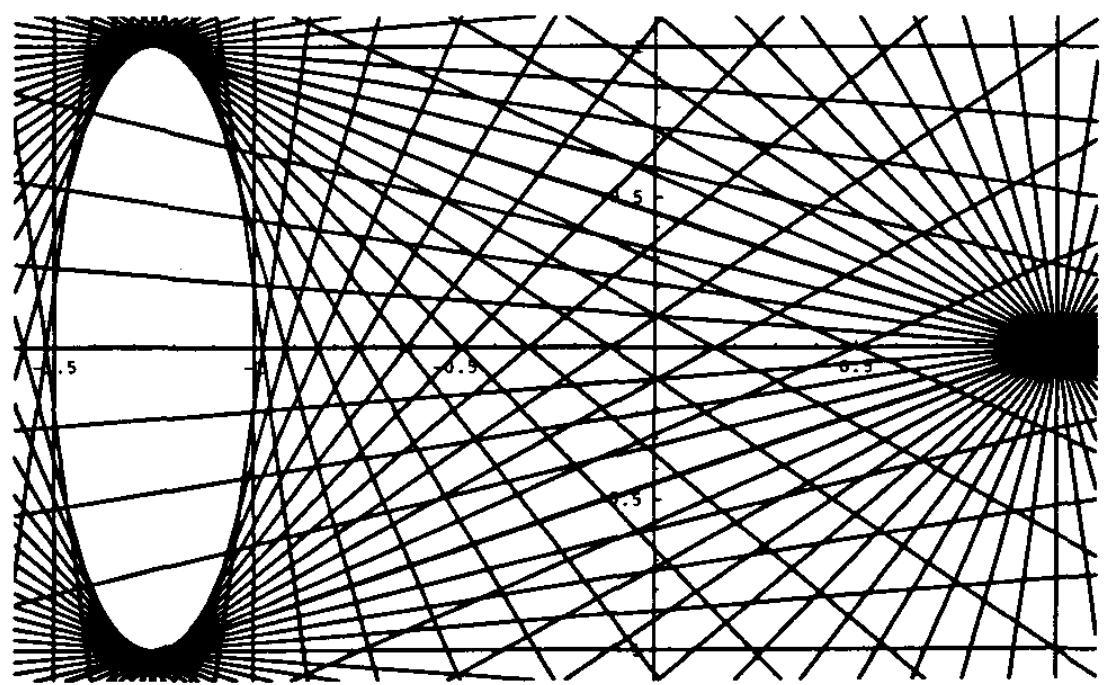

$$
\mathbf{A}=\left(\left(\begin{array}{ccc}
1 & 0 & 0 \\
0 & -1 & 0 \\
0 & 0 & -3 / 2
\end{array}\right),\left(\begin{array}{lll}
0 & 0 & 0 \\
0 & 0 & 1 \\
0 & 1 & 0
\end{array}\right)\right)
$$

FIGURE 2. 


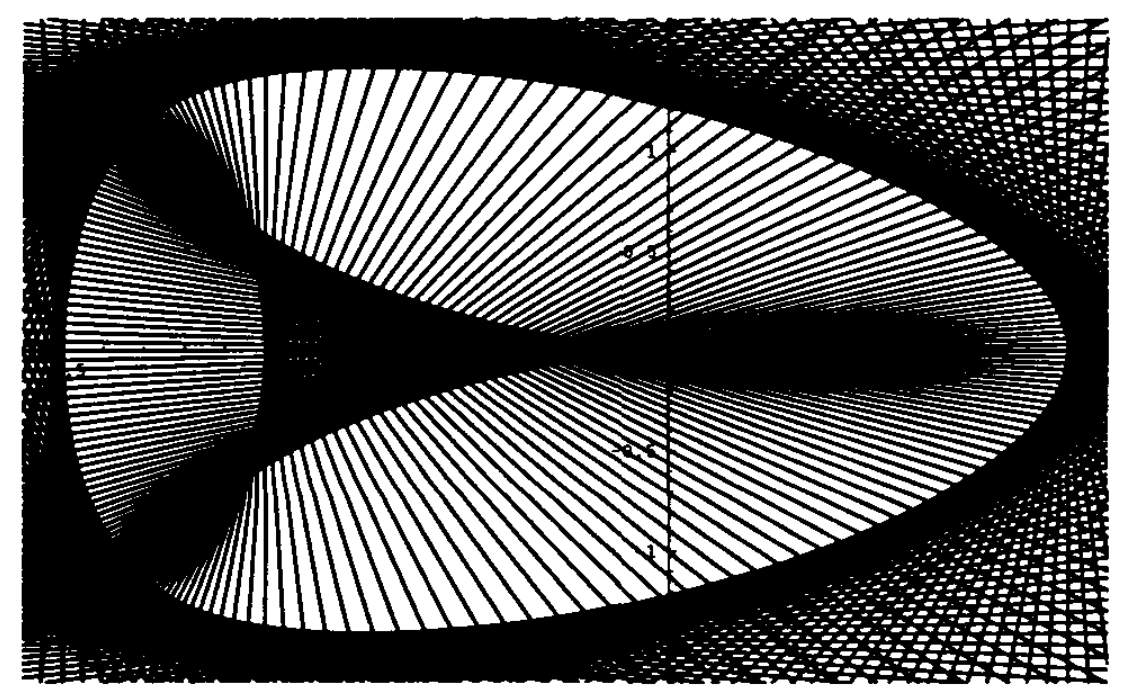

$$
\mathbf{A}=\left(\left(\begin{array}{ccc}
1 & 0 & 0 \\
0 & -1 & 0 \\
0 & 0 & -3 / 2
\end{array}\right),\left(\begin{array}{lll}
0 & 0 & 1 \\
0 & 0 & 1 \\
1 & 1 & 0
\end{array}\right)\right)
$$

FIGURE 3.

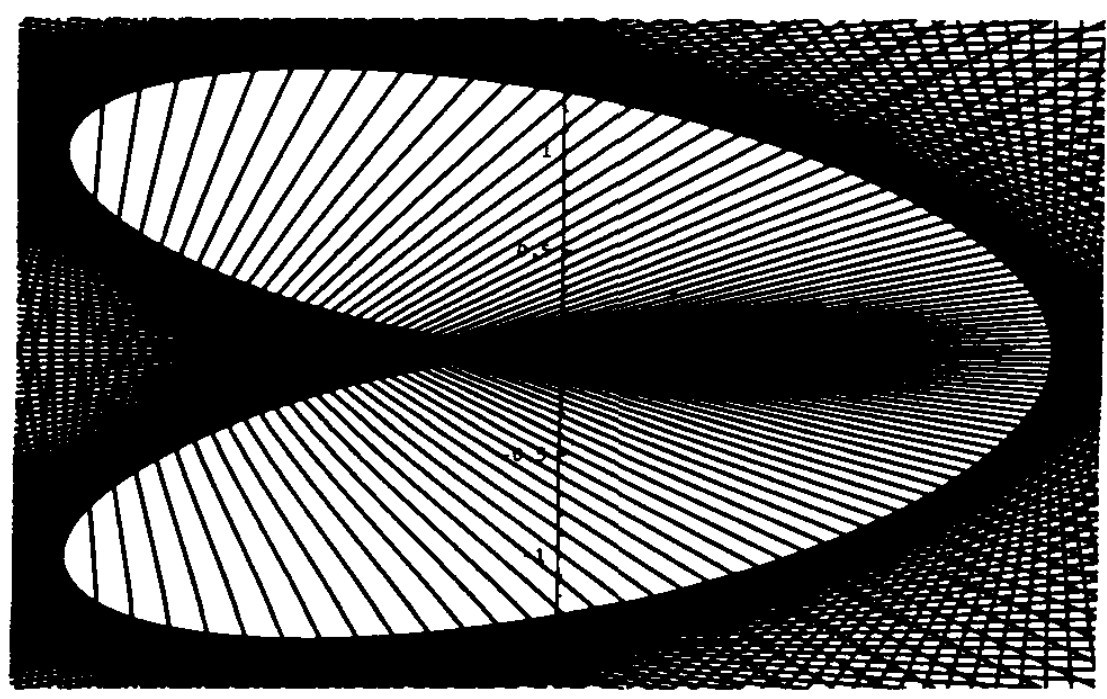

$$
\mathbf{A}=\left(\left(\begin{array}{ccc}
1 & 0 & 0 \\
0 & -1 & 0 \\
0 & 0 & -1
\end{array}\right),\left(\begin{array}{lll}
0 & 0 & 1 \\
0 & 0 & 1 \\
1 & 1 & 0
\end{array}\right)\right)
$$

FIGURE 4. 


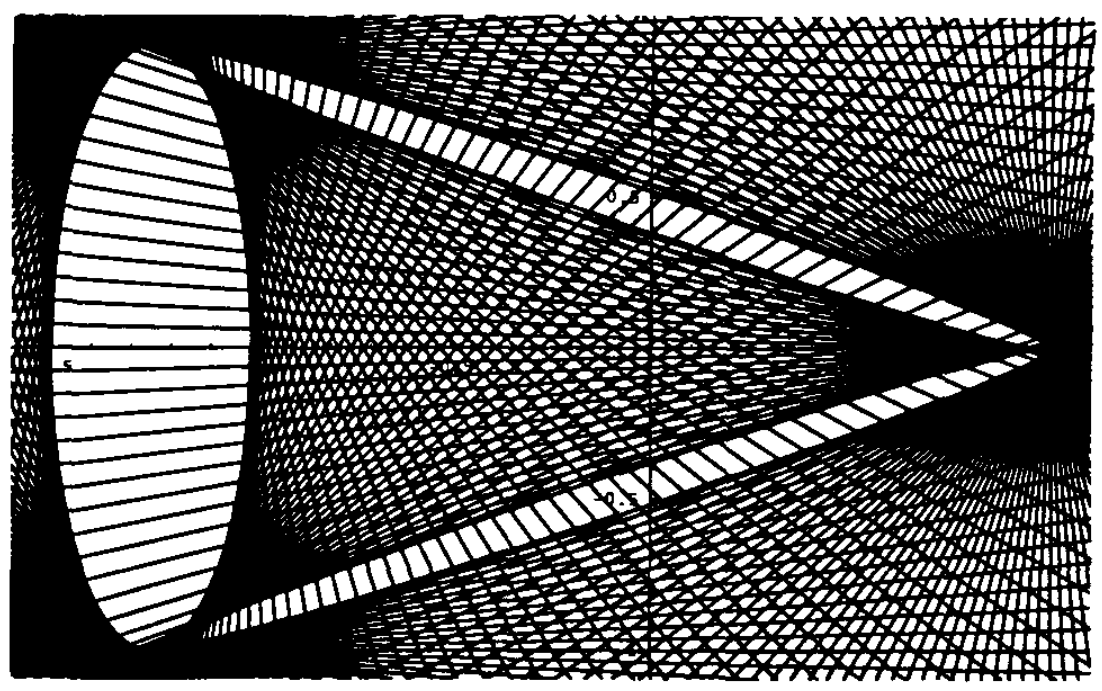

$$
\mathbf{A}=\left(\left(\begin{array}{ccc}
1 & 0 & 0 \\
0 & -1 & 0 \\
0 & 0 & -3 / 2
\end{array}\right),\left(\begin{array}{ccc}
0 & 0 & 1 / 10 \\
0 & 0 & 1 \\
1 / 10 & 1 & 0
\end{array}\right)\right)
$$

FIGURE 5.

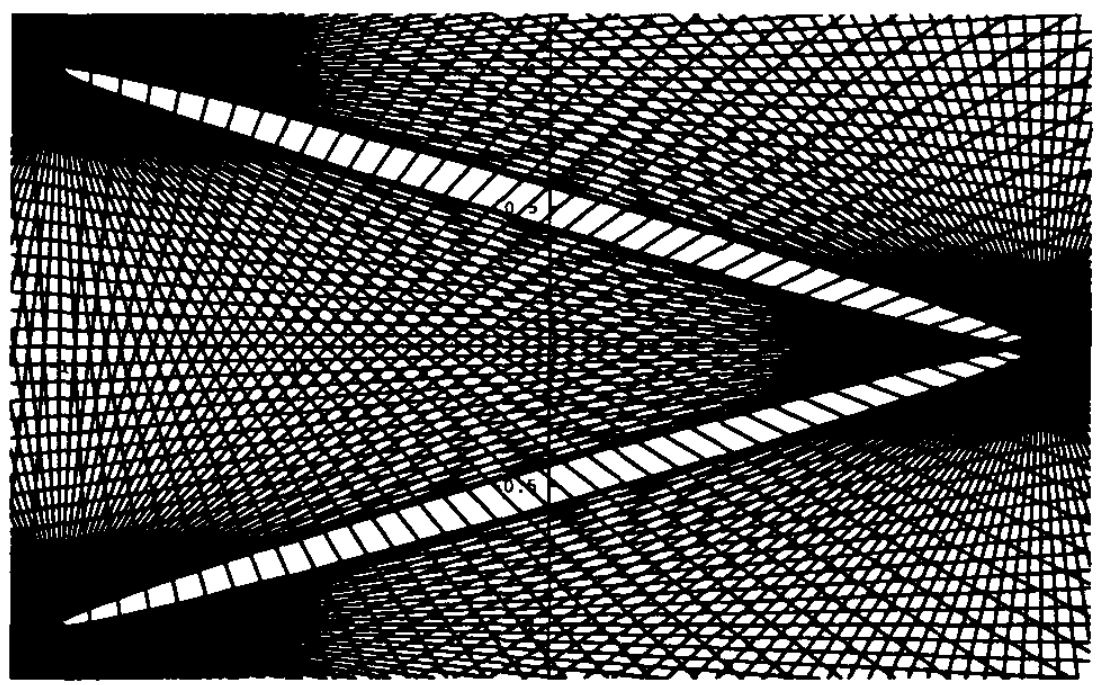

$$
\mathbf{A}=\left(\left(\begin{array}{ccc}
1 & 0 & 0 \\
0 & -1 & 0 \\
0 & 0 & -1
\end{array}\right),\left(\begin{array}{ccc}
0 & 0 & 1 / 10 \\
0 & 0 & 1 \\
1 / 10 & 1 & 0
\end{array}\right)\right)
$$

FiguRE 6. 
mental solution $E(x, t)$ of the hyperbolic differential operator

$$
\operatorname{det}\left(I \frac{\partial}{\partial t}+A_{1} \frac{\partial}{\partial x_{1}}+A_{2} \frac{\partial}{\partial x_{2}}\right)
$$

at $t=1$ is the numerical range $W(A)$ [4, Theorem 7.7]. The triangular regions inside $W(A)$ in Figures 2-6 correspond to Petrovsky lacunas [3, Example 10.6], so $E(x, t)$ is a homogeneous polynomial of degree $n-3=0$ there, that is, a nonzero constant function. Because the matrix valued distribution $\mathscr{W}_{\mathbf{A}}$ can be expressed in terms of derivatives of $E$, it vanishes in these regions.

A further illustration for a pair of $(7 \times 7)$ hermitian matrices associated with the linearised Lundquist equations of magnetogasdynamics is given in [7, Figures 1a-b]. The numerical ranges of certain matrices are displayed in a fashion similar to that above in [13, Figures 1-9, pages 139-147]. Unfortunately, the interior structure of the numerical range is omitted from these diagrams.

\section{Proof of Theorem 6.4}

We first show that $\mathbf{R}(A) \subseteq \gamma(\mathbf{A})^{c}$. Let $x \in \mathbf{R}(A)$. We must find an open neighbourhood $U$ of $(0, x)$ in $\mathbb{R}^{3}$ such that the function

$$
(\epsilon, y) \mapsto G_{y+\epsilon e_{0}}(\mathbf{A}), \quad(\epsilon, y) \in U \backslash\left(\{0\} \times \mathbb{R}^{2}\right)
$$

is the restriction to $U \backslash\left(\{0\} \times \mathbb{R}^{2}\right)$ of a continuous function defined in $U$. Then by Painlevé's Theorem [9, Theorem 10.6], $G_{\omega}(\mathbf{A})$ is monogenic in a neighbourhood of $(0, x)$, because $G_{\omega}(\mathbf{A})$ is monogenic above and below $\{0\} \times \mathbb{R}^{2}$. Hence $x \in \gamma(\mathbf{A})^{c}$.

We start by examining the plane wave decomposition (9). Let $y \in \mathbb{R}^{2}$ and set $\mathbf{B}(y)=y I-\mathbf{A}$. First, we convert the integral (9) to a contour integral

$$
\int_{\mathbb{T}}(\langle\mathbf{B}(y), s\rangle-\epsilon s I)^{-2} d \mu(s)=-i \int_{\mathbb{T}}(\langle\mathbf{B}(y), s(z)\rangle-\epsilon s(z) I)^{-2} z^{-1} d z
$$

for the function $s: \mathbb{C} \backslash\{0\} \rightarrow \mathbb{C}^{2}$ defined by (16). The integral (20) may be evaluated using Cauchy's Residue Theorem by finding the residues of the function

$$
z \mapsto(\langle\mathbf{B}(y), z s(z)\rangle-\epsilon z s(z) I)^{-2} z^{-1}
$$

in the open unit disk $D=\{z \in \mathbb{C}|| z \mid<1\}$. The formula (10) holds for any $s \in \mathbb{T}$ and $\epsilon \neq 0$. We split the integral (20) accordingly into its scalar part belonging to the linear subspace $\left\{T e_{0} \mid T \in \mathscr{L}\left(\mathbb{C}^{n}\right)\right\}$ of $\mathscr{L}\left(\mathbb{C}^{n}\right)_{(2)}$ and its vector part belonging to the linear subspace $\left\{T_{1} e_{1}+T_{2} e_{2} \mid T_{1}, T_{2} \in \mathscr{L}\left(\mathbb{C}^{n}\right)\right\}$ of $\mathscr{L}\left(\mathbb{C}^{n}\right)_{(2)}$. There is no component belonging to the linear subspace $\left\{T e_{1} e_{2} \mid T \in \mathscr{L}\left(\mathbb{C}^{n}\right)\right\}$ of $\mathscr{L}\left(\mathbb{C}^{n}\right)_{(2)}$. 
We make a few observations. If the limit of the scalar part

$$
-i \int_{\mathbb{T}}\left(\langle\mathbf{B}(y), s\rangle^{2}-\epsilon^{2} I\right)\left(\langle\mathbf{B}(y), s\rangle^{2}+\epsilon^{2} I\right)^{-2} z^{-1} d z
$$

of the integral (20) exists in $\mathscr{L}\left(\mathbb{C}^{n}\right)$ and is nonzero as $\epsilon \rightarrow 0$, then by formula (9), the Cauchy kernel $G_{y+\epsilon e_{0}}(\mathbf{A})$ has a jump discontinuity at $y \in \mathbb{R}^{2}$ as $\epsilon \rightarrow 0$. In this case $y \in \gamma(\mathbf{A})$. The formula

$$
\mathscr{W}_{\mathbf{A}}(\phi)=\lim _{\varepsilon \rightarrow 0^{+}} \int_{\mathbb{R}^{2}}\left[G_{y+\varepsilon e_{0}}(\mathbf{A})-G_{y-\varepsilon e_{0}}(\mathbf{A})\right] \phi(y) d y, \quad \phi \in C_{c}^{\infty}\left(\mathbb{R}^{2}\right),
$$

mentioned in the proof of $[17$, Theorem 6.2$]$ shows that the jump

$$
y \mapsto \lim _{\varepsilon \rightarrow 0^{+}}\left[G_{y+\varepsilon e_{0}}(\mathbf{A})-G_{y-\varepsilon e_{0}}(\mathbf{A})\right]
$$

where it exists, is the Schwartz kernel of the matrix valued distribution $\mathscr{W}_{\mathrm{A}}$. The vector part

$$
\frac{i|\epsilon|}{4 \pi^{2}} \int_{\mathbb{T}} s\langle\mathbf{B}(y), s\rangle\left(\langle\mathbf{B}(y), s\rangle^{2}+\epsilon^{2} I\right)^{-2} z^{-1} d z
$$

of the integral (20) depends only on $|\epsilon|$ for $\epsilon \neq 0$, so the vector part of

$$
G_{y+\varepsilon e_{0}}(\mathbf{A})-G_{y-\varepsilon e_{0}}(\mathbf{A})
$$

is zero for all $\varepsilon>0$, in accordance with the fact that the distribution $\mathscr{W}_{\mathrm{A}}$ takes its values in the subspace $\mathscr{L}\left(\mathbb{C}^{n}\right)$ of $\mathscr{L}\left(\mathbb{C}^{n}\right)_{(2)}$.

The strategy used to prove that $x \in \gamma(\mathbf{A})^{c}$ is to show that the matrix-valued integral (22) converges to zero as $\epsilon \rightarrow 0+$, whereas the integral (23) converges in $\mathscr{L}\left(\mathbb{C}^{n}\right)_{(2)}$ uniformly for all $y \in \mathbb{R}^{2}$ in a neighbourhood of $x$.

We first examine the residues of the matrix-valued integrand

$$
z \mapsto\left(\langle\mathbf{B}(y), s(z)\rangle^{2}-\epsilon^{2} I\right)\left(\langle\mathbf{B}(y), s(z)\rangle^{2}+\epsilon^{2} I\right)^{-2} z^{-1}
$$

of (22). Note that if $y$ belongs to an open neighbourhood of $x$ in $\mathbf{R}(A) \subset \rho(A)$, then the point $z=0$ is a removable singularity, for $B_{1}(y)+i B_{2}(y)=\left(y_{1}+i y_{2}\right) I-\left(A_{1}+i A_{2}\right)$ is invertible and we may write $\left(\langle\mathbf{B}(y), s(z)\rangle^{2}-\epsilon^{2} I\right)\left(\langle\mathbf{B}(y), s(z)\rangle^{2}+\epsilon^{2} I\right)^{-2} z^{-1}$ as

$$
\left(\langle\mathbf{B}(y), z s(z)\rangle^{2}-z \epsilon^{2} I\right)\left(\langle\mathbf{B}(y), z s(z)\rangle^{2}+z \epsilon^{2} I\right)^{-2} z,
$$

where $\left(\langle\mathbf{B}(y), z s(z)\rangle^{2}-z \epsilon^{2} I\right)\left(\langle\mathbf{B}(y), z s(z)\rangle^{2}+z \epsilon^{2} I\right)^{-2} \rightarrow 4\left(B_{1}(y)+i B_{2}(y)\right)^{-2}$ as $z \rightarrow 0$. 
LEMMA 9.1. Let $\epsilon>0$. If $z \neq 0$ is a solution of $\operatorname{det}(\langle\mathbf{B}(y), s(z)\rangle+i \epsilon I)=0$, then $\bar{z}^{-1}$ satisfies $\operatorname{det}\left(\left\langle\mathbf{B}(y), s\left(\bar{z}^{-1}\right)\right\rangle-i \epsilon I\right)=0$. In particular, if $\phi$ is the function defined in Corollary 7.8 , then $\phi(-\epsilon, y)=\overline{\phi(\epsilon, y)}^{-1}$.

Proof. The identity $\left\langle\mathbf{B}(y), s\left(\bar{z}^{-1}\right)\right\rangle=\langle\mathbf{B}(y), s(z)\rangle^{*}$ holds because $A_{1}$ and $A_{2}$ are hermitian matrices, so

$$
\begin{aligned}
\operatorname{det}\left(\left\langle\mathbf{B}(y), s\left(\bar{z}^{-1}\right)\right\rangle-i \epsilon I\right) & =\operatorname{det}\left(\langle\mathbf{B}(y), s(z)\rangle^{*}-i \epsilon I\right) \\
& =\overline{\operatorname{det}(\langle\mathbf{B}(y), s(z)\rangle+i \epsilon I) .}
\end{aligned}
$$

Let $\lambda_{1, x}: V_{\zeta} \rightarrow \mathbb{C}$ and $\phi: U_{x} \rightarrow \mathbb{C}$ be the functions defined in Corollary 7.8. Then $z \mapsto \overline{\lambda_{1, x}\left(\bar{z}^{-1}\right)}, z \in V_{\zeta}$ is analytic and equal to $\lambda_{1, x}$ on $V_{\zeta} \cap \mathbb{T}$ where $\lambda_{1, x}$ has real values. By analytic continuation, it follows that $\overline{\lambda_{1, x}\left(\bar{z}^{-1}\right)}=\lambda_{1, x}(z)$ for all $z \in V_{\zeta}$. According to the definition of $\phi$ we have $\lambda_{1, x}\left(\overline{\phi(\epsilon, y)^{-1}}\right)=\overline{\lambda_{1, x}(\phi(\epsilon, y))}=-i \epsilon$ and $\lambda_{1, x}(\phi(-\epsilon, y))=-i \epsilon$. The uniqueness of $\phi$ ensures that $\phi(-\epsilon, y)=\overline{\phi(\epsilon, y)}^{-1}$ for all $(\epsilon, y) \in U_{x}$

Hence, solutions $z \in \mathbb{C} \backslash\{0\}$ of

$$
\operatorname{det}\left(\langle\mathbf{B}(y), s(z)\rangle^{2}+\epsilon^{2} I\right)=\operatorname{det}(\langle\mathbf{B}(y), s(z)\rangle+i \epsilon I) \operatorname{det}(\langle\mathbf{B}(y), s(z)\rangle-i \epsilon I)=0
$$

either satisfy $z \in \mathbb{T}$ (if $\epsilon=0$ ) or come in pairs $z=\xi$ and $z=\bar{\xi}^{-1}$, one inside the open unit disk $D$ and the other outside the closed unit disk $\bar{D}$.

The following representation was obtained in [7, Equation (4.4a)] using a plane wave decomposition different to the one used here.

LEMma 9.2. Suppose that $x \in \mathbb{R}^{2} \backslash \sigma(A)$ does not belong to the Kippenhahn curves $C(A)$. Then there exists an open neighbourhood $U$ of $x$ in $\mathbb{R}^{2}$ disjoint from $C(A)$ and two contours, $\Gamma_{1}(x)$ surrounding $\bar{D}$ and $\Gamma_{2}(x)$ contained in $D$, both anticlockwise oriented, such that $\langle\mathbf{B}(y), s(z)\rangle$ is invertible in $\mathscr{L}\left(\mathbb{C}^{n}\right)$ for all $z \in \Gamma_{1}(x) \cup \Gamma_{2}(x)$ and $y \in U$, and the limit

$$
\begin{aligned}
\lim _{\epsilon \rightarrow 0+} & \int_{\mathbb{J}}\left(\langle\mathbf{B}(y), s(z)\rangle^{2}-\epsilon^{2} I\right)\left(\langle\mathbf{B}(y), s(z)\rangle^{2}+\epsilon^{2} I\right)^{-2} z^{-1} d z \\
& =\frac{1}{2} \lim _{\epsilon \rightarrow 0} \int_{\Gamma_{1}(x)+\Gamma_{2}(x)}\left(\langle\mathbf{B}(y), s(z)\rangle^{2}-\epsilon^{2} I\right)\left(\langle\mathbf{B}(y), s(z)\rangle^{2}+\epsilon^{2} I\right)^{-2} z^{-1} d z \\
& =\frac{1}{2} \int_{\Gamma_{1}(x)+\Gamma_{2}(x)}\langle y I-\mathbf{A}, s(z)\rangle^{-2} z^{-1} d z,
\end{aligned}
$$

exists and the convergence is uniform for all $y \in U$. 
Proof. Suppose that $\zeta \in \bar{D}$ satisfies

$$
\operatorname{det}(\langle x I-\mathbf{A}, s(\zeta)\rangle)=0
$$

If $\zeta \in \mathbb{T}$, then we know that an analytic parametrization (18) exists in an open neighbourhood $V_{\zeta}$ of $\zeta$ in $\mathbb{C}$ for which $\lambda_{1, x}(\zeta)=0$. By assumption, $x \in \mathbb{R}^{2} \backslash C(A)$, so Corollary 7.8 implies that there exists a smooth function $y \mapsto \phi(0, y)$ defined in a neighbourhood $U$ of $x$ in $\mathbb{R}^{2}$ disjoint from $C(A)$, such that $\phi(0, x)=\zeta, \phi(0, y) \in \mathbb{T}$ and $\lambda_{1, y}(\phi(0, y))=0$ for all $y \in U$.

Furthermore, the solution $\phi(\xi, y)$ of $\lambda_{1, y}(\phi(\xi, y))=i \xi$ is a smooth function for $(\xi, y)$ in a neighbourhood of $(0, x)$ in $\mathbb{R}^{3}$. Hence, given any contours $\Gamma_{1}(x)$ and $\Gamma_{2}(x)$ satisfying the conditions above, there exists an open neighbourhood $V$ of $(0, x) \in \mathbb{R}^{3}$ such that $\phi( \pm \epsilon, y)$ lies in the region between the contours $\Gamma_{1}(x)$ and $\Gamma_{2}(x)$ for all ( $\pm \epsilon, y) \in V$. According to Corollary 7.8, the complex numbers $\phi( \pm \epsilon, y)$ are distinct and both converge to $\phi(0, y)$ as $\epsilon \rightarrow 0+$.

On the other hand, $X_{x}=\{z \in D \mid \operatorname{det}(\langle x I-\mathbf{A}, s(z)\rangle)=0\}$ is a finite subset $\left\langle\zeta_{j}\right\rangle_{j=1}^{k}$ of the open unit disk $D$. We claim that there exists an open neighbourhood $W$ of $(0, x)$ and disjoint closed disks $D_{j} \subset D$ centred at $\zeta_{j} \in X_{x}$ such that for every $(\xi, y) \in W$, all solutions $z$ of the equation

$$
\operatorname{det}(\langle y I-\mathbf{A}, s(z)\rangle+i \xi I)=0
$$

lie in the union $\cup_{j=1}^{k} D_{j}$ of the disjoint closed disks.

This would again follow from Corollary 7.8 if we knew that an analytic parametrization (18) exists in an open neighbourhood $V_{\zeta}$ of $\zeta \in X_{x}$. We have already noted that, except for a finite set of points, such an analytic parametrization is possible [5, Theorem 3.3.12]. More simply, setting $\mathbf{B}(y)=y I-\mathbf{A},(27)$ can be written as

$$
\begin{aligned}
& \operatorname{det}(\langle y I-\mathbf{A}, s(z)\rangle+i \xi I) \\
&=(2 z)^{-n} \operatorname{det}(\langle\mathbf{B}(y), 2 z s(z))+i 2 z \xi I) \\
&=(2 z)^{-n} \operatorname{det}\left(B_{1}(y)-i B_{2}(y)\right) \operatorname{det}\left(z^{2} A I\right. \\
&\left.-\left(B_{1}(y)-i B_{2}(y)\right)^{-1}\left(B_{1}(y)+i B_{2}(y)\right)+i 2 z \xi\left(B_{1}(y)-i B_{2}(y)\right)^{-1}\right) \\
&=(2 z)^{-n} \operatorname{det}\left(B_{1}(y)-i B_{2}(y)\right) \operatorname{det}\left[\left(z I+i \xi\left(B_{1}(y)-i B_{2}(y)\right)^{-1}\right)^{2}\right. \\
&\left.-\left(B_{1}(y)-i B_{2}(y)\right)^{-1}\left(B_{1}(y)+i B_{2}(y)\right)+\xi^{2}\left(B_{1}(y)-i B_{2}(y)\right)^{-2}\right] \\
&= 0,
\end{aligned}
$$

provided that $y \in \rho(A)$. By assumption $x \in \rho(A)$, so the equation is valid for all $y$ in a neighbourhood of $x$ and the solutions $z$ of (27) can be expressed in terms of the eigenvalues of an $(n \times n)$ matrix depending continuously on $(\xi, y)$. The unordered $n$-tuple of eigenvalues of this matrix valued function depends continuously on the 
parameters $(\xi, y)$ [22, Theorem II.5.1] facilitating the construction of the required disjoint closed disks $D_{j}, j=1, \ldots, k$.

According to Lemma 9.1, poles of the function (24) come in pairs $\left(z, \bar{z}^{-1}\right)$ lying either inside the open unit disk $D$ or outside the closed unit disk $\bar{D}$ for all $y$ in a neighbourhood of $x$. Now choose the inner contour $\Gamma_{2}(x)$ to surround every closed disk $D_{j}$, and choose $\Gamma_{1}(x)$ to lie between $\mathbb{T}$ and points $\bar{z}^{-1}, z \in \cup_{j=1}^{k} D_{j}$, outside $\bar{D}$. Next choose the intersection of all open neighbourhoods $V$ of $(0, x)$ corresponding to the finitely many solutions $\zeta \in \mathbb{T}$ of (26) and take the intersection $V^{\prime}$ of this open set with the open set $W$ corresponding to the finitely many solutions $\zeta_{j} \in D, j=1, \ldots, k$ of (27).

Then for every $(\epsilon, y) \in V^{\prime}$, the contour integral

$$
\int_{\Gamma_{1}(x)+\Gamma_{2}(x)}\left(\langle\mathbf{B}(y), s(z)\rangle^{2}-\epsilon^{2} I\right)\left(\langle\mathbf{B}(y), s(z)\rangle^{2}+\epsilon^{2} I\right)^{-2} z^{-1} d z
$$

is $2 \pi i$ times the sum of the residues of the integrand at the distinct poles $\phi( \pm \epsilon, y)$ and $4 \pi i$ times the sum of the residues at poles near solutions $\zeta \in D$ of (26), because both contours $\Gamma_{1}(x)$ and $\Gamma_{2}(x)$ surround these. The possibility of a pole at zero in the case that $y \in \sigma(A)$ is excluded.

The function

$$
(\epsilon, y) \mapsto \int_{\Gamma_{1}(x)+\Gamma_{2}(x)}\left(\langle\mathbf{B}(y), s(z)\rangle^{2}-\epsilon^{2} I\right)\left(\langle\mathbf{B}(y), s(z)\rangle^{2}+\epsilon^{2} I\right)^{-2} z^{-1} d z
$$

is continuous on $V^{\prime}$, so equality (25b) is immediate. To prove equality (25a), we need to look separately at those poles $\zeta$ of (24) satisfying (26) lying inside the open disk $D$ and those lying on $\mathrm{T}$.

The sum of the residues of the function (24) belonging to $\cup_{j=1}^{k} D_{j} \subset D$ is equal to

$$
\frac{1}{2 \pi i} \int_{\Gamma_{2}(x)}\left(\langle\mathbf{B}(y), s(z)\rangle^{2}-\epsilon^{2} I\right)\left(\langle\mathbf{B}(y), s(z)\rangle^{2}+\epsilon^{2} I\right)^{-2} z^{-1} d z
$$

for all $(\epsilon, y) \in V^{\prime}$, so this is uniformly continuous in $(\epsilon, y) \in V^{\prime}$.

Now we need to show that the sum of the residues of the function (24) over all the poles $\phi( \pm \epsilon, y)$ converges uniformly in $y$ as $\epsilon \rightarrow 0+$ to twice the sum of the residues of $z \mapsto\langle\mathbf{B}(y), s(z)\rangle^{-2} z^{-1}$ over all the poles $\phi(0, y)$. According to Lemma 9.1, one of the poles $\phi( \pm \epsilon, y)$ lies in $D$ and the other is outside $D$, so then equality (25a) will be established.

The set $Z_{y}$ of all solutions $\zeta \in \mathbb{T}$ of (26), is finite for each $y$ in a neighbourhood of $x$, so it suffices to prove that each residue of $(24)$ at $\phi( \pm \epsilon, y)$ converges uniformly to the residue of $z \mapsto\langle\mathbf{B}(y), s(z)\rangle^{-2} z^{-1}$ at $\phi(0, y)$.

For every solution $\zeta \in Z_{y}$, there exists a neighbourhood $V_{\zeta}$ in $\mathbb{C}$ such that $V_{\zeta} \cap\left(Z_{y}\right)$ $\{\zeta\})=\emptyset$ and the parametrization (18) holds. Then, writing $\lambda_{j}$ for the eigenvalues 
$\lambda_{j, y}(z)$ of $\langle y I-\mathbf{A}, s(z)\rangle$ in (18), the equality

(28) $\left(\langle\mathbf{B}(y), s(z)\rangle^{2}-\epsilon^{2} I\right)\left(\langle\mathbf{B}(y), s(z)\rangle^{2}+\epsilon^{2} I\right)^{-2} z^{-1}=\sum_{j=1}^{m} \frac{\lambda_{j}(z)^{2}-\epsilon^{2}}{\left(\lambda_{j}(z)^{2}+\epsilon^{2}\right)^{2} z} P_{j}(z)$

holds for all $z \in V_{\zeta}$.

By assumption, the eigenvalue functions $\lambda_{j}$ have at most one zero, $z=\zeta$, in $V_{\zeta}$. We may suppose that for some integer $k, 1 \leq k \leq m$, we have $\lambda_{1}(\zeta)=\cdots=\lambda_{k}(\zeta)=0$ and $\lambda_{j}(\zeta) \neq 0$ for $j>k$. The terms in the sum (28) corresponding to the latter are analytic in the open set $V_{\zeta}$.

By Corollary 7.8, there exists a neighbourhood $U_{x}$ of $(0, x)$ in $\mathbb{R}^{3}$ such that for all $j$ with $1 \leq j \leq k, \phi_{j}(\epsilon, y)=\lambda_{j}^{-1}(i \epsilon)$ defines a $C^{\infty}$-function on $U_{x}$ satisfying $\lambda_{j}^{\prime}\left(\phi_{j}(\epsilon, y)\right) \neq 0$ for all $(\epsilon, y) \in U_{x}$. In particular, the set of all $y \in \mathbb{R}^{2}$ such that $(\xi, y) \in U_{x}$ for some $\xi \in \mathbb{R}$, is disjoint from $C(A)$. Then for $\epsilon>0$, we have

$$
\begin{aligned}
\operatorname{Res}( & \left.\frac{\lambda_{j}(z)^{2}-\epsilon^{2}}{\left(\lambda_{j}(z)^{2}+\epsilon^{2}\right)^{2} z} P_{j}(z) ; \phi_{j}(\epsilon, y)\right) \\
= & \frac{1}{\lambda_{j}^{\prime}\left(\phi_{j}(\epsilon, y)\right)^{2}}\left[\frac{d}{d z}\left(\frac{\lambda_{j}(z)^{2}-\epsilon^{2}}{\left(\lambda_{j}(z)+i \epsilon\right)^{2} z} P_{j}(z)\right)\right]_{\phi_{j}(\epsilon, y)} \\
& \quad-\frac{\lambda_{j}^{\prime \prime}\left(\phi_{j}(\epsilon, y)\right) P_{j}\left(\phi_{j}(\epsilon, y)\right)}{\lambda_{j}^{\prime}\left(\phi_{j}(\epsilon, y)\right)^{3} \phi_{j}(\epsilon, y)} .
\end{aligned}
$$

Here we have written $\left(\lambda_{j}(z)^{2}+\epsilon^{2}\right)^{2}=\left(\lambda_{j}(z)+i \epsilon\right)^{2}\left(\lambda_{j}(z)-i \epsilon\right)^{2}$ and noted that

$$
\left(\lambda_{j}(z)-i \epsilon\right)^{2}=\left(\frac{\lambda_{j}(z)-\lambda_{j}\left(\phi_{j}(\epsilon, y)\right)}{z-\phi_{j}(\epsilon, y)}\right)^{2}\left(z-\phi_{j}(\epsilon, y)\right)^{2}
$$

gives rise to a pole of order two at $\phi_{j}(\epsilon, y)$. Now

$$
\frac{d}{d z} \frac{\lambda_{j}^{2}-\epsilon^{2}}{\left(\lambda_{j}+i \epsilon\right)^{2}}=2 \lambda_{j}^{\prime} \frac{\lambda_{j}\left(\lambda_{j}+i \epsilon\right)-\left(\lambda_{j}^{2}-\epsilon^{2}\right)}{\left(\lambda_{j}+i \epsilon\right)^{3}}=2 i \epsilon \lambda_{j}^{\prime} \frac{\lambda_{j}-i \epsilon}{\left(\lambda_{j}+i \epsilon\right)^{3}}
$$

is zero at $\phi_{j}(\epsilon, y)$. According to Corollary 7.8 , the function $(\epsilon, y) \mapsto \lambda_{j}^{\prime}\left(\phi_{j}(\epsilon, y)\right)$ is $C^{\infty}$ and nonzero in a neighbourhood of $(0, x)$. It follows that the matrix

$$
\begin{aligned}
\operatorname{Res} & \left(\frac{\lambda_{j}(z)^{2}-\epsilon^{2}}{\left(\lambda_{j}(z)^{2}+\epsilon^{2}\right)^{2} z} P_{j}(z) ; \phi_{j}(\epsilon, y)\right) \\
& =\frac{1}{2 \lambda_{j}^{\prime}\left(\phi_{j}(\epsilon, y)\right)^{2}}\left[\frac{d}{d z} \frac{P_{j}(z)}{z}\right]_{\phi_{j}(\epsilon, y)}-\frac{\lambda_{j}^{\prime \prime}\left(\phi_{j}(\epsilon, y)\right) P_{j}\left(\phi_{j}(\epsilon, y)\right)}{2 \lambda_{j}^{\prime}\left(\phi_{j}(\epsilon, y)\right)^{3} \phi_{j}(\epsilon, y)} .
\end{aligned}
$$

converges uniformly for all $y$ in a neighbourhood of $x$ as $\epsilon \rightarrow 0^{+}$. 
The residue at each of the poles $\phi_{j}( \pm \epsilon, y)$ contributes to the integral over $\Gamma_{1}(x)+$ $\Gamma_{2}(x)$, so in the limit, we obtain twice the sum of the residues of the matrix-valued function $z \mapsto\langle\mathbf{B}(y), s(z)\rangle^{-2} z^{-1}$ at poles $\zeta \in \mathbb{T}$ and inside $\mathbb{T}$. We have proved the required formula.

The next lemma establishes that the scalar component of $\omega \mapsto G_{\omega}(\mathbf{A})$ is continuous in a neighbourhood of $x \in \mathbf{R}(A)$ in $\mathbb{R}^{3}$.

LEMMA 9.3. For every $x \in \mathbf{R}(A)$ the matrix valued function

$$
y \mapsto G_{y+\epsilon e_{0}}(\mathbf{A})-G_{y-\epsilon e_{0}}(\mathbf{A}), \quad y \in \mathbb{R}^{2},
$$

converges to zero as $\epsilon \rightarrow 0+$, uniformly for all $y$ in a neighbourhood of $x$.

Proof. By Corollary 7.8, $x \in C(A)^{c}$ and $\mathbf{R}(A) \subseteq \rho(A)$, so the representation of Lemma 9.2 is valid. But there are no poles interior to $\mathbb{T}$ or exterior to $\mathbb{T}$. Hence, the integral over $\Gamma_{2}(x)$ is zero and we can deform $\Gamma_{1}(x)$ to $\infty$. It follows that integral $(25 b)$ is zero.

The following argument treats the residues of the integrand

$$
z \mapsto s(z)\langle\mathbf{B}(y), s(z)\rangle\left(\langle\mathbf{B}(y), s(z)\rangle^{2}+\epsilon^{2} I\right)^{-2} z^{-1}
$$

of the contour integral (23), the vector part of the Cauchy kernel.

Let $x \in \mathbb{R}^{2} \backslash(C(A) \cup \sigma(A))$. As in the proof of Lemma 9.2, there exists an open set $V_{\zeta} \subset D$ about each solution $\zeta \in D$ of $\operatorname{det}(\langle x I-\mathbf{A}, s(z)\rangle)=0$ and a neighbourhood $W$ of $(0, x)$ in $\mathbb{R}^{3}$, such that for every $(\xi, y) \in W$, all solutions $z$ of the equation $\operatorname{det}(\langle y I-\mathbf{A}, s(z)\rangle-i \xi I)=0$ belong to $\cup_{\zeta} V_{\zeta}$. Moreover, the closures of the open sets $V_{\zeta}$ are pairwise disjoint.

The sum $R_{\zeta}(\epsilon, y)$ of the residues of the function (30) at poles in $V_{\zeta}$ is a continuous function of $(\epsilon, y)$, because it can be represented as a contour integral of the continuous function (30) over a contour inside the open unit disk $D$ surrounding $V_{\zeta}$. Then

$$
\lim _{\epsilon \rightarrow 0+} \epsilon R_{\zeta}(\epsilon, y)=0
$$

uniformly for $y$ in a neighbourhood of $x$.

Now let $\zeta \in \mathbb{T}$ be a solution of $\operatorname{det}(\langle x I-\mathbf{A}, s(z)\rangle)=0$. Suppose that $j, 1 \leq$ $j \leq m$, is an index for which $\lambda_{j}(\zeta)=0$ and $\phi(\epsilon, y)=\lambda_{j}^{-1}(i \epsilon)$ lies in $D$ for all $0<\epsilon<\delta$, otherwise, replace $i \epsilon$ by $-i \epsilon$. Such a solution exists by Corollary 7.8 and the assumption that $x \in \mathbb{R}^{2} \backslash C(A)$. Furthermore, $(\epsilon, y) \mapsto \phi(\epsilon, y)$ is $C^{\infty}$ in a neighbourhood of $(0, x)$ and $\left|\lambda_{j}^{\prime}(\phi(\epsilon, y))\right|$ is bounded below. 
LEMMA 9.4. Let $x \in \mathbb{R}^{2} \backslash C(A)$ and suppose that $\phi(\epsilon, y)$ is a pole of $(30)$ belonging to the open unit disk $D$, as defined above. Then

$$
\epsilon \operatorname{Res}\left(\frac{s(z)\langle\mathbf{B}(y), s(z)\rangle\left(\langle\mathbf{B}(y), s(z)\rangle^{2}+\epsilon^{2} I\right)^{-2}}{z} ; \phi(\epsilon, y)\right)
$$

converges as $\epsilon \rightarrow 0+$, uniformly for all $y$ in a neighbourhood of $x$.

PROOF. As in the proof of Lemma 9.2, it suffices to prove that

$$
\epsilon \operatorname{Res}\left(\frac{s(z) \lambda_{j}(z)}{\left(\lambda_{j}(z)^{2}+\epsilon^{2}\right)^{2} z} P_{j}(z) ; \phi(\epsilon, y)\right)
$$

converges as $\epsilon \rightarrow 0+$ uniformly for $y$ in a neighbourhood of $x$.

By assumption, $\phi(\epsilon, y)=\lambda_{j}^{-1}(i \epsilon)$ lies in the open unit disk $D$ for all $0<\epsilon<\delta$. Then

$$
\begin{aligned}
\epsilon \operatorname{Res} & \left(\frac{s(z) \lambda_{j}(z)}{\left(\lambda_{j}(z)^{2}+\epsilon^{2}\right)^{2} z} P_{j}(z) ; \phi(\epsilon, y)\right) \\
= & \frac{\epsilon}{\lambda_{j}^{\prime}(\phi(\epsilon, y))^{2}}\left[\frac{d}{d z}\left(\frac{s(z) \lambda_{j}(z)}{\left(\lambda_{j}(z)+i \epsilon\right)^{2} z} P_{j}(z)\right)\right]_{\phi(\epsilon, y)} \\
& +\frac{i \lambda_{j}^{\prime \prime}(\phi(\epsilon, y)) s(\phi(\epsilon, y)) P_{j}(\phi(\epsilon, y))}{4 \lambda_{j}^{\prime}(\phi(\epsilon, y))^{3} \phi(\epsilon, y)}
\end{aligned}
$$

Note that

$$
\frac{d}{d z} \frac{\lambda_{j}}{\left(\lambda_{j}+i \epsilon\right)^{2}}=\lambda_{j}^{\prime} \frac{\left(\lambda_{j}+i \epsilon\right)-2 \lambda_{j}}{\left(\lambda_{j}+i \epsilon\right)^{3}}=-\lambda_{j}^{\prime} \frac{\lambda_{j}-i \epsilon}{\left(\lambda_{j}+i \epsilon\right)^{3}}
$$

is zero at $\phi(\epsilon, y)$. On the other hand,

$$
\frac{\epsilon \lambda_{j}(\phi(\epsilon, y))}{\left(\lambda_{j}(\phi(\epsilon, y))+i \epsilon\right)^{2}}\left[\frac{d}{d z}\left(\frac{s(z)}{z} P_{j}(z)\right)\right]_{\phi(\epsilon, y)}=-\frac{i}{4}\left[\frac{d}{d z}\left(\frac{s(z)}{z} P_{j}(z)\right)\right]_{\phi(\epsilon, y)}
$$

and the other terms in the residue formula converge uniformly for $y$ in a neighbourhood of $x$ as $\epsilon \rightarrow 0+$.

Consequently, for every $x \in \mathbf{R}(A)$, the matrix-valued integral (22) converges to zero as $\epsilon \rightarrow 0$, whereas the integral (23) converges in $\mathscr{L}\left(\mathbb{C}^{n}\right)$ uniformly in a neighbourhood of $x$. The Cauchy kernel $\omega \mapsto G_{\omega}(\mathbf{A})$ is therefore continuous in a neighbourhood of $(0, x)$ in $\mathbb{R}^{3}$, proving that $x \in \gamma(\mathbf{A})^{c}$.

To complete the proof of Theorem 6.4, it still remains to prove that $x \in \gamma(\mathbf{A})$ for all $x \in \mathbb{R}^{2} \backslash \mathbf{R}(A)$. We essentially follow the somewhat abbreviated proof of [7, Theorem 4.3] after noting that Condition II of [7, Theorem 4.3] is superfluous by 
appealing to our Lemma 7.1. As mentioned in [7, page 316], the proof is based on a closely related argument of Petrovsky [27, page 348].

Let $\Delta(\mathbf{A})$ be the set of all $x \in \mathbb{R}^{2} \backslash C(A)$ such that $\lim _{\varepsilon \rightarrow 0^{+}}\left[G_{y+\varepsilon e_{0}}(\mathbf{A})-G_{y-\varepsilon e_{0}}(\mathbf{A})\right]$ converges uniformly to zero for all $y$ in an open neighbourhood of $x$ disjoint from $C(A)$. Then $\Delta(\mathbf{A})$ is an open subset of $\mathbb{R}^{2}$ containing $\gamma(\mathbf{A})^{c}$, because for every $x \in \gamma(\mathbf{A})^{c}$, the Cauchy kernel $\omega \mapsto G_{\omega}(\mathbf{A})$ is continuous for every $\omega$ in a neighbourhood of $(0, x)$ in $\mathbb{R}^{3}$.

Suppose that

$$
\left(\mathbb{R}^{2} \backslash(\overline{\mathbf{R}(A)} \cup C(A))\right) \cap \Delta(\mathbf{A}) \neq \emptyset .
$$

We shall obtain a contradiction from the assumption (31), so showing that

$$
\mathbb{R}^{2} \backslash(\overline{\mathbf{R}(A)} \cup C(A)) \subseteq \Delta(\mathbf{A})^{c} \subset \gamma(\mathbf{A})
$$

Because

$$
\left(\left(\mathbb{R}^{2} \backslash(\overline{\mathbf{R}(A)} \cup C(A))\right) \cap \Delta(\mathbf{A})\right) \backslash \sigma(A)
$$

is a nonempty open set, there exists a nonempty open subset $U$ of the set (32) such that $\lim _{\varepsilon \rightarrow 0^{+}}\left[G_{y+\varepsilon e_{0}}(\mathbf{A})-G_{y-\varepsilon e_{0}}(\mathbf{A})\right]$ converges uniformly to zero for all $y \in U$.

Now $U$ is disjoint from $\mathbf{R}(A)$ and $\sigma(A)$. If for every $x \in U$, every pole of the function

$$
z \mapsto\langle x I-\mathbf{A}, s(z)\rangle^{-1}
$$

lies on $\mathbb{T}$, then $U \subset \mathbf{R}(A)$. By Lemma 9.1, poles $z \notin \mathbb{T}$ of (33) come in pairs $z \in D$ and $\bar{z}^{-1} \in \bar{D}^{c}$, so there must exist $x \in U$ such that (33) has a pole inside $D$. Moreover, by the argument of Lemma 9.2, the set $\left\{y \in \mathbb{R}^{2} \mid \sigma\left(\left((y I-\tilde{A})^{*}\right)^{-1}(y I-\tilde{A})\right) \cap D \neq \emptyset\right\}$ is an open subset of $\mathbb{R}^{2}$, so for every $y$ belonging to some neighbourhood of $x$, the function $z \mapsto\langle y I-\mathbf{A}, s(z)\rangle^{-1}$ has poles inside $D$. By shrinking $U$ if necessary, we suppose that $U$ has this property.

Then the calculation of the residues in Lemmas 9.2 and 9.4 is still valid because $U$ is disjoint from both $\sigma(A)$ and $C(A)$. By Lemma 9.2, the limit

$$
\begin{gathered}
\lim _{\varepsilon \rightarrow 0} \int_{\pi}\left(\langle y I-\mathbf{A}, s\rangle^{2}-\varepsilon^{2} I\right)\left(\langle y I-\mathbf{A}, s\rangle^{2}+\varepsilon^{2} I\right)^{-2} d \mu(s) \\
=-\frac{i}{2} \int_{\Gamma_{1}(x)+\Gamma_{2}(x)}\langle y I-\mathbf{A}, s(z)\rangle^{-2} z^{-1} d z
\end{gathered}
$$

is a matrix-valued real analytic function for all $y$ in a neighbourhood $U_{x}$ of $x$ contained in $U$-a constant times the function

$$
y \mapsto \lim _{\varepsilon \rightarrow 0^{+}}\left[G_{y+\varepsilon \varepsilon_{0}}(\mathbf{A})-G_{y-\varepsilon e_{0}}(\mathbf{A})\right], \quad y \in U_{x} .
$$


By assumption, $U_{x} \subset \Delta(\mathbf{A})$, so for all $y \in U_{x}$, we have

$$
\int_{\Gamma_{1}(x)+\Gamma_{2}(x)}\langle y I-\mathbf{A}, s(z)\rangle^{-2} z^{-1} d z=0 .
$$

The point $z=0$ is a removable singularity of the integrand in equation (34) because $y \in U_{x} \subset \rho(A)$.

Up until this point, we have worked locally with solutions $z=\phi(y)$ of the equation $\operatorname{det}(\langle y I-\mathbf{A}, s(z)\rangle)=0$ for $\phi(y)$ belonging to a neighbourhood of $\mathbb{T}$.

Now let us consider all solutions $z=\phi(y) \in \mathbb{C}$ of the simultaneous equations

$$
\begin{array}{r}
\operatorname{det}(\mu I-\langle\mathbf{A}, s(z)\rangle)=0, \\
\mu-\langle y, s(z)\rangle=0,
\end{array}
$$

for $y \in \mathbb{R}^{2}$.

For $z \neq 0$, equation (35) is equivalent to $\operatorname{det}(z \mu I-\langle\mathbf{A}, z s(z)\rangle)=0$ and the function $(\mu, z) \mapsto \operatorname{det}(z \mu-\langle\mathbf{A}, z s(z)\rangle)$ is a polynomial in two variables. Equation (35) therefore determines an algebraic function $z \mu(z)$ of $z$ [1, Chapter 8, Definition 2]. Except for a finite set $\Sigma$ of points in $\mathbb{C}$, each function element $(\mu, \Omega)$ of $\mu$ can be continued along any arc not passing through one of the exceptional points belonging to $\Sigma$ [1, page 294]. It follows from Rellich's Theorem and equation (18) that $\Sigma$ is disjoint from $\mathbb{T}$.

Suppose that $\left(\mu_{j}, \Omega_{j}\right)$ is a function element of $\mu$ such that $\Omega_{j}$ is disjoint from $\Sigma \cup\{0\}$. Then $\zeta \mapsto\left(s_{1}(\zeta): s_{2}(\zeta):-\mu_{j}(\zeta)\right), \zeta \in \Omega_{j}$, is a smooth local parametrization of the algebraic curve $C(A)^{*}$ of Section 7. If $y \in \mathbb{R}^{2}$ and $z \in \mathbb{C}$ satisfy (36) for $\mu=\mu_{j}(z)$, and $\mu_{j}^{\prime}(z)-\langle y, s\rangle^{\prime}(z)=0$, then by Lemma 7.1, $y \in C(A)$. Consequently, if $y \notin C(A)$, then any solution $z_{0}$ of (35)-(36) with $\mu=\mu_{j}(z)$ has the property that

$$
\mu_{j}^{\prime}\left(z_{0}\right)-\langle y, s\rangle^{\prime}\left(z_{0}\right) \neq 0 \text {. }
$$

Suppose that $y \notin C(A)$. By the remark after Lemma 7.6, there exists an open neighbourhood $V_{y}$ of $y$ in $\mathbb{R}^{2}$ and an analytic function $w \mapsto \phi_{j}(w), w \in V_{y}$, of two real variables such that $\left\langle w, s\left(\phi_{j}(w)\right)\right\rangle=\mu_{j}\left(\phi_{j}(w)\right)$ for all $w \in V_{y}$. Hence,

$$
\operatorname{det}\left(\left\langle w, s\left(\phi_{j}(w)\right)\right\rangle I-\left\langle\mathbf{A}, s\left(\phi_{j}(w)\right)\right\rangle\right)=0
$$

and for every $w \in V_{y}$ the complex number $\phi_{j}(w)$ is a pole of the function

$$
z \mapsto\langle w I-\mathbf{A}, s(z)\rangle^{-1} .
$$

Now according to (31), we are assuming that poles of function (33) exist inside $D$. So there exist a nonzero integer $k$ and $2 k$ functions $y \mapsto \pm \phi_{j}(y), j=1, \ldots, k$, 
defined for $y \in U_{x}$, that are analytic in two real variables and poles of (33) belonging to $D$. We can also assume that they have the property that $\pm \phi_{j}(y) \notin \Sigma \cup\{0\}$ for all $y \in U_{x}$ and that they are constructed, as above, from the algebraic function $z \mu(z)$.

This is valid, because to any nonzero exceptional point $z \in \Sigma$, there corresponds a unique solution $y \in \mathbb{R}^{2}$ of (36) satisfying the equations

$$
y_{1} s_{1}(z)+y_{2} s_{2}(z)=\mu y_{1} s_{1}(\bar{z})-y_{2} s_{2}(\bar{z})=\bar{\mu} .
$$

Here we use the observation that $s_{1}(z) s_{2}(\bar{z})+s_{1}(\bar{z}) s_{2}(z)=0$ if and only if $|z|=1$ and $\Sigma$ is disjoint from $\mathbb{T}$. The point $z=0$ is associated with points $\zeta \in \sigma(A)$ with $y_{1}+i y_{2}=\zeta$ and $\lim _{z \rightarrow 0} z \mu_{j}(z)=\zeta / 2$, for some function element $\left(\mu_{j}, \Omega_{j}\right)$ of $\mu$ with $0 \in \bar{\Omega}_{j}$.

With these preliminary observations out of the way, we will obtain a contradiction from the assumption that (34) holds in a neighbourhood $U_{x}$ of $x$.

Let $x_{1} \in \mathbf{R}(A)$ and suppose that $t \mapsto \gamma(t), 0 \leq t \leq 1$, is a smooth curve in $\mathbb{R}^{2}$ such that $\gamma(0)=x$ and $\gamma(1)=x_{1}$. Suppose further that where $\gamma$ crosses a curve belonging to $C(A)$, it does so nontangentially and avoids all intersections, cusps and isolated points. This is possible because there are only finitely many such points. Furthermore, we suppose that $\gamma$ also avoids the image in $\mathbb{R}^{2}$ of the exceptional points $\Sigma$ and the spectrum $\sigma(A)$ of $A$. Then in a neighbourhood of any point in $\gamma([0,1])$, the functions $\left\{\phi_{j}\right\}_{j=1}^{k}$ defined by the algebraic function $z \mu(z)$ from (35)-(36) in the manner described above, do not take values in $\Sigma \cup\{0\}$. Moreover, we have $\phi_{j}(\gamma(1)) \in \mathbb{T}$ and $\phi_{j}(\gamma(0)) \in U_{x} \subset D$ for $j=1, \ldots, k$. Let

$$
t_{0}=\sup \left\{t>0: \phi_{j}(\gamma(s)) \in D \text { for every } 0 \leq s \leq t \text { and } j=1, \ldots, k\right\} .
$$

Then $0<t_{0} \leq 1$ and, by continuity, for some $m=1, \ldots, k$, we must have $\phi_{m}\left(\gamma\left(t_{0}\right)\right) \in$ T. If $\mu_{j}^{\prime}\left(\phi_{m}\left(\gamma\left(t_{0}\right)\right)\right)-\left\langle\gamma\left(t_{0}\right), s\right\rangle^{\prime}\left(\phi_{m}\left(\gamma\left(t_{0}\right)\right)\right) \neq 0$, then by Rellich's Theorem and Lemma 7.6, there exists $\delta>0$ such that $\phi_{m}(\gamma(t)) \in \mathbb{T}$ for all $t \in\left(t_{0}-\delta, t_{0}+\delta\right)$, contradicting the definition of $t_{0}$. Hence $\gamma\left(t_{0}\right) \in C(A)$ by Proposition 7.7.

According to our assumption, equation (34), the sum $\operatorname{Res}(y)$ of the residues of the function $z \mapsto\langle y I-\mathbf{A}, s(z)\rangle^{-2} z^{-1}$ at $\pm \phi_{j}(y)$ and $\pm \overline{\phi_{j}}(y)^{-1}, j=1, \ldots, k$, is zero for all $y \in U_{x}$. The outer integral about the contour $\Gamma_{1}(x)$ in (34) surrounds $\pm \overline{\phi_{j}}(y)^{-1}$ and the integral is calculated from the residues at $\pm \overline{\phi_{j}}(y)^{-1}$ by the Cauchy integral formula.

For each $0<t<t_{0}$, there exist contours $\Gamma_{1}(\gamma(t)) \subset \bar{D}^{c}$ and $\Gamma_{2}(\gamma(t)) \subset D$ and neighbourhoods $V_{\gamma(t)}$ of $\gamma(t)$ such that $\Gamma_{1}(\gamma(t))$ surrounds $\left\{\overline{\phi_{j}}(y)^{-1}\right\}_{j=1}^{k}$ and $\Gamma_{2}(\gamma(t))$ surrounds $\left\{\phi_{j}(y)\right\}_{j=1}^{k}$ for all $y \in V_{\gamma(t)}$, and the contours do not surround any other poles of function (33) for any $y \in V_{\gamma(t)}$.

To see that this construction is possible, suppose that $\phi_{\ell}$ is some other distinct solution of simultaneous equations (35)-(36) such that $\zeta=\phi_{\ell}(\gamma(t))=\phi_{1}(\gamma(t)) \in D$, 
say, for some $0<t<t_{0}$. Then $\langle\gamma(t), s(\zeta)\rangle=\mu_{\ell}(\zeta)=\mu_{1}(\zeta)$ for two eigenvalues $\mu_{\ell}(z)$ and $\mu_{1}(z)$ of the matrix $\langle\mathbf{A}, s(z)\rangle$, for all $z \in \mathbb{C}$ in a neighbourhood of $\zeta$. Then $\zeta$ must be a branch point of the eigenvalues of the matrix valued function $z \mapsto\langle\mathbf{A}, s(z)\rangle$, that is, $\zeta \in \Sigma$. This contradicts our choice of the arc $\gamma$. Hence, all solutions of simultaneous equations (35)-(36) have distinct values at each point of $\gamma$. By continuity, for each $0 \leq t<t_{0}$ we can choose a neighbourhood $V_{\gamma(t)}$ of $\gamma(t)$ in which solutions of (35)-(36) have this property and contours $\Gamma_{1}(\gamma(t))$ and $\Gamma_{2}(\gamma(t))$ with the properties described above.

Then the function

$$
\operatorname{Res}(y)=\frac{1}{2 \pi i} \int_{\Gamma_{1}(y(t))+\Gamma_{2}(y(t))}\langle y I-\mathbf{A}, s(z)\rangle^{-2} z^{-1} d z
$$

defined for all $y \in V_{y(t)}$ and $0 \leq t<t_{0}$ agrees on $U_{x} \cap V_{x}$ with the sum $\operatorname{Res}(y)$ of residues defined above for $y \in U_{x}$. Clearly, $\operatorname{Res}(y)$ is an analytic function of the two real variables $y$, so by analytic continuation, $\operatorname{Res}(\gamma(t))=0$ for all $0 \leq t<t_{0}$.

The point $\phi_{m}\left(\gamma\left(t_{0}\right)\right) \in \mathbb{T}$ corresponds to where $\gamma$ crosses the curve $C(A)$ at $t_{0}$ with $\phi_{m}\left(\gamma\left(t_{0}\right)\right)$ the direction of the unit normal. As mentioned above, $\gamma$ may have crossed a curve in $C(A)$ earlier, leading to the appearance of poles of the function (33) additional to $\left\{\phi_{j}(y)\right\}_{j=1}^{k}$ for $y \in V_{\gamma(t)}$, but the chosen contours do not surround these.

Because $\gamma$ avoids all intersections, isolated points and cusps, for each $j=1, \ldots, k$ with $j \neq m$, we have $\phi_{j}\left(\gamma\left(t_{0}\right)\right) \neq \phi_{m}\left(\gamma\left(t_{0}\right)\right)$ and $\phi_{j}(\gamma(t))$ is bounded away from T for all $0 \leq t \leq t_{0}$ (the unit normal is unique). Any other poles $\phi\left(\gamma\left(t_{0}\right)\right)$ of (33) are not associated with function elements of $\boldsymbol{\mu}$ at which (19) holds for $y=\gamma\left(t_{0}\right)$. Otherwise, by Proposition 7.7, $\gamma\left(t_{0}\right)$ would lie on the intersection of curves belonging to $C(A)$ with $\phi\left(\gamma\left(t_{0}\right)\right) \in \mathbb{T}$, the unit normal to one of the curves.

However, it is impossible that $\operatorname{Res}(\gamma(t))=0$ for all $0 \leq t<t_{0}$, because the residues diverge at $\phi_{m}\left(\gamma\left(t_{0}\right)\right) \in \mathbb{T}$, but are uniformly bounded at $\phi_{j}(\gamma(t)), 0 \leq t \leq t_{0}$ for $j \neq m$. This follows from an asymptotic analysis of (29) as $y \rightarrow \gamma\left(t_{0}\right)$ along $\gamma$. The asymptotic analysis is facilitated by the fact that $\mu_{j}$ and $P_{j}$ are analytic in a neighbourhood of $\phi_{m}\left(\gamma\left(t_{0}\right)\right)$ by Rellich's theorem. Rather than repeat the calculation here, see [7, Equation (4.24)], and the references there that follow that equation. The original assumption that $\operatorname{Res}(y)=0$ for all $y$ in a neighbourhood $U_{x}$ of $x$ must be false, so that (31) is false. We have shown the inclusion $\mathbb{R}^{2} \backslash(\overline{\mathbf{R}(A)} \cup C(A)) \subset \gamma(\mathbf{A})$.

If $x \in \mathbf{R}(A)^{c} \cap \overline{\mathbf{R}(A)}=\partial \mathbf{R}(A)$, then by Corollary 7.9, $x$ is an element of $C(A)$, so it only remains to treat the case $x \in C(A)$. In this case, the asymptotic analysis mentioned above ensures that we can actually make $\lim _{\varepsilon \rightarrow 0^{+}}\left[G_{y+\varepsilon e_{0}}(\mathrm{~A})-G_{y-\varepsilon e_{0}}(\mathrm{~A})\right]$ diverge as $y \rightarrow x$ in some direction in $C(A)^{c}$, namely, from the direction into which the curvature vector points, proving that $x \in \gamma(\mathbf{A})$. We have established the inclusion $\mathbb{R}^{2} \backslash \mathbf{R}(A) \subseteq \gamma(\mathbf{A})$. 


\section{Further developments}

In this section, we consider which of the preceding arguments are applicable to a more general situation. A plane wave decomposition formula similar to formula (9) has been obtained for a $d$-tuple $\mathbf{A}=\left(A_{1}, \ldots, A_{d}\right)$ of bounded linear operators acting on a Banach space $X$ just under the assumption that the spectrum $\sigma(\langle\xi, \mathbf{A}\rangle)$ of the bounded linear operator $\langle\xi, \mathbf{A}\rangle=\sum_{j=1}^{d} \xi_{j} A_{j}$ is real for each $\xi \in \mathbb{R}^{d}[18$, Lemma 2.5]. Actually, the plane wave decomposition is adopted as the definition of the Cauchy kernel $G_{(\cdot)}(\mathbf{A})$ off $\mathbb{R}^{d}$ in the case that the Weyl calculus does not exist, that is, when the necessary exponential bounds for the $d$-tuple $\mathbf{A}$ of operators fail. It agrees with the power series expansion [18, Equation (5)] outside a sufficiently large ball and it agrees with the Cauchy-Stieltjes transform of the Weyl functional calculus when this exists [17, Equation (5)]. The same formula will work for a $d$-tuple $\mathbf{A}=\left(A_{1}, \ldots, A_{d}\right)$ of unbounded linear operators if the closure $\overline{\langle\xi, \mathbf{A}\rangle}$ of $\langle\xi, \mathbf{A}\rangle$ is densely defined and has real spectrum for each $\xi \in \mathbb{R}^{d}$.

The monogenic spectrum $\gamma(\mathbf{A})$ of the $d$-tuple $\mathbf{A}$ is the set of singularities of the Cauchy kernel $G_{(\cdot)}(\mathrm{A})$, that is to say, $\gamma(\mathrm{A})$ is the complement of the largest set in $\mathbb{R}^{d+1}$ on which $\omega \mapsto G_{\omega}(\mathbf{A})$ is a monogenic function with values in a Clifford module $\mathscr{L}(X)_{(d)}$ over the bounded linear operators on the Banach space $X$.

In the Hilbert space setting with $d=2$ and $\mathbf{A}=\left(A_{1}, A_{2}\right)$ bounded selfadjoint operators, Definition 6.1 and the proof of Proposition 6.2 still make sense, so that $\mathbf{R}(A)^{c}$ is contained in the numerical range $W(A)$ of the bounded linear operator $A=A_{1}+i A_{2}$. The convex hull of $\gamma(\mathbf{A})$ is equal to the numerical range $W(A)$. However, we have no information about the relationship of $\mathbf{R}(A)$ and $\gamma(\mathbf{A})$ in the infinite dimensional setting.

If we assume only that $\mathbf{A}=\left(A_{1}, A_{2}\right)$ is a pair of matrices such that $\sigma(\langle\xi, \mathbf{A}\rangle)$ is real for each $\xi \in \mathbb{R}^{2}$, then we can no longer appeal to Rellich's Theorem, crucial to the proofs of Sections 7 and 9. A pair of simultaneously triangularisable matrices, each with real spectrum, falls into this category-such matrices need not be hermitian. Moreover, the algebraic function $z \mu(z)$ defined in Section 9 may now have a finite number of singularities on the unit circle $\mathbb{T}$.

Rather than use the Rellich formula (18), we could attempt to express the matrix

$$
\langle y I-\mathbf{A}, s(z)\rangle
$$

in terms of the resolvent $(\mu I-\langle\mathbf{A}, s(z)\rangle)^{-1}, \mu \in \rho(\langle\mathbf{A}, s(z)\rangle)$, of $\langle\mathbf{A}, s(z)\rangle$ for suitable $z \in \mathbb{C}$. The Cauchy kernel $G_{(\cdot)}(\mathbf{A})$ of $\mathbf{A}$ can also be expressed in terms of resolvent operators $(\mu I-\langle\mathbf{A}, s\rangle)^{-1}$ by means of the plane wave decomposition. This approach has the advantage of being applicable to a $d$-tuple of linear operators acting in a Banach space. The preceding analysis applies to pair of matrices satisfying the spectral condition just mentioned, although singularities of the algebraic function 
$z \mu(z)$ defined in Section 9 on the unit circle $\mathbb{T}$ must now be accounted for. A similar remark was made in [7, Footnote 1]. The observation that a $d$-tuple $\mathbf{A}$ of matrices with the property that $\sigma(\langle\xi, \mathbf{A}\rangle)$ is real for each $\xi \in \mathbb{R}^{d}$ has a Weyl functional calculus is proved in [16] by operator-theoretic methods.

Finally, we note that for the triple $\sigma=\left(\sigma_{1}, \sigma_{2}, \sigma_{3}\right)$ of $(2 \times 2)$ Pauli matrices, the monogenic spectrum $\gamma(\sigma)$ of $\sigma$ is the unit sphere $\mathbb{S}$ in $\mathbb{R}^{3}[2$, Theorem 4.1]. Points exterior to $\mathbb{S}$ belong to infinitely many tangent planes to $\mathbb{S}$, but points inside $\mathbb{S}$ have none. Nevertheless, the interior of $\mathbb{S}$ is a lacuna of $\gamma(\sigma)$ corresponding to the fundamental solution of Weyl's equation. The simple equality $\gamma(\mathbf{A})=\mathbf{R}(A)^{c}$, suitably interpreted, does not go over to higher order systems $\mathbf{A}=\left(A_{1}, \ldots, A_{d}\right)$, $d>2$, without some additional connection between $d$ and the size of the matrices $A_{1}, \ldots, A_{d}$.

\section{References}

[1] L. Ahlfors, Complex analysis, 2nd Edition (McGraw Hill, New York, 1966).

[2] R. F. V. Anderson, 'The Weyl functional calculus', J. Funct. Anal. 4 (1969), 240-267.

[3] M. Atiyah, R. Bott and L. Gårding, 'Lacunas for hyperbolic differential operators with constant coefficients I', Acta Math. 124 (1970), 109-189.

[4] —-, 'Lacunas for hyperbolic differential operators with constant coefficients II', Acta Math. 131 (1973), 145-206.

[5] H. Baumgärtel, Analytic perturbation theory for matrices and operators, Operator Theory: Advances and Applications 15 (Birkhäuser, Basel, 1985).

[6] J. Bazer and D. H. Y. Yen, 'The Riemann matrix of $(2+1)$-dimensional symmetric hyperbolic systems', Comm. Pure Appl. Math. 20 (1967), 329-363.

[7] _ 'Lacunas of the Riemann matrix of symmetric-hyperbolic systems in two space variables', Comm. Pure Appl. Math. 22 (1969), 279-333.

[8] J. Bochnak, M. Coste and M.-F. Roy, Géométrie algébrique réelle (Springer, New York, 1987).

[9] F. Brackx, R. Delanghe and F. Sommen, Clifford analysis, Research Notes in Mathematics 76 (Pitman, Boston, 1982).

[10] H. Bremermann, Distributions, complex variables and Fourier transforms (Addison-Wesley, Reading, 1964).

[11] N. Dunford and J. T. Schwartz, Linear operators. Part II, (Interscience, New York, 1963).

[12] G. Greiner and W. Ricker, 'Commutativity of compact selfadjoint operators', Studia Math. 112 (1995), 109-125.

[13] K. Gustafson and D. K. M. Rao, Numerical range, the field of values of linear operators and matrices (Springer, New York, 1997).

[14] J. A. Hillman, B. Jefferies, W. Ricker and B. Straub, 'Differential properties of the numerical range map of pairs of matrices', Linear Algebra Appl. 267 (1997), 317-334.

[15] B. Jefferies, 'The Weyl calculus for hermitian matrices', Proc. Amer. Math. Soc. 124 (1996), 121-128.

[16] - 'Exponential bounds for noncommuting systems of matrices', Studia Math. 144 (2001), 197-207.

[17] B. Jefferies and A. McIntosh, 'The Weyl calculus and Clifford analysis', Bull. Austral. Math. Soc. 57 (1998), 329-341. 
[18] B. Jefferies, A. McIntosh and J. Picton-Warlow, 'The monogenic functional calculus', Studia Math. 136 (1999), 99-119.

[19] B. Jefferies and W. Ricker, 'Commutativity for $(2 \times 2)$ systems of selfadjoint matrices', Linear and Multilinear Algebra 35 (1993), 107-114.

[20] F. John, Plane waves and spherical means applied to partial differential equations (Interscience, New York, 1955).

[21] M. Joswig and B. Straub, 'On the numerical range map', J. Austral. Math. Soc. (Series A) 65 (1998), 267-283.

[22] T. Kato, Perturbation theory for linear operators, 2nd Edition (Springer, Berlin, 1980).

[23] R. Kippenhahn, 'Über den Wertevorrat einer Matrix’, Math. Nachr. 6 (1951), 193-228.

[24] C. Li, A. McIntosh and T. Qian, 'Clifford algebras, Fourier transforms and singular convolution operators on Lipschitz surfaces', Rev. Mat. Iberoamericana 10 (1994), 665-721.

[25] F. D. Murnaghan, 'On the field of values of a square matrix', Proc. Nat. Acad. Sci. U.S.A. 18 (1932), 246-248.

[26] E. Nelson, 'Operants: A functional calculus for non-commuting operators', in: Functional analysis and related fields, Proceedings of a conference in honour of Professor Marshal Stone, Univ. of Chicago, May 1968 (ed. F. E. Browder) (Springer, Berlin, 1970) pp. 172-187.

[27] I. Petrovsky, 'On the diffusion of waves and lacunas for hyperbolic equations', Mat. Sbornik 17 (1945), 289-368.

[28] F. Rellich, 'Störungstheorie der Spektralzerlegung I', Math. Ann. 113 (1937), 600-619.

[29] W. Ricker, 'The Weyl calculus, joint spectra and commutativity of compact, selfadjoint operators', Integral Equations Operator Theory 22 (1995), 333-338.

[30] J. Ryan, 'Plemelj formulae and transformations associated to plane wave decompositions in complex Clifford analysis', Proc. London Math. Soc. 64 (1992), $70-94$.

[31] I. R. Shafarevich, Basic algebraic geometry (Springer, New York, 1977).

[32] F. Sommen, 'Plane wave decompositions of monogenic functions', Ann. Polon. Math. 49 (1988), 101-114.

[33] M. E. Taylor, 'Functions of several self-adjoint operators', Proc. Amer. Math. Soc. 19 (1968), 91-98.

[34] V. A. Vassiliev, Ramified integrals, singularities and lacunas, Mathematics and its Applications 315 (Kluwer, Dordrecht, 1995).

[35] H. Weitzner, 'Green's function for two-dimensional magnetohydrodynamic waves, I, II', Phys. Fluids 4 (1961), 1238-1245, 1246-1250.

\section{School of Mathematics}

The University of New South Wales

Sydney NSW 2052

Australia

e-mail: b.jefferies@unsw.edu.au,bernd@maths.unsw.edu.au 